\title{
Functional comparison of MERS-coronavirus lineages reveals increased zoonotic potential of the recombinant lineage 5
}

\section{Simon Schroeder}

Charité-Universitätsmedizin Berlin

\section{Christin Mache}

Robert Koch Institut

\section{Hannah Kleine-Weber}

German Primate Center

\section{Doreen Muth}

Charité-Universitätsmedizin Berlin

\section{Victor Corman}

Charité-Universitätsmedizin Berlin, corporate member of Freie Universit\&\#x00E4,t Berlin, HumboldtUniversit\&\#x00E4,t zu Berlin, and Berlin Institute of Health, Institute of Virology https://orcid.org/00000002-3605-0136

\section{Diana Fatykhova}

Charité-Universitätsmedizin Berlin

\section{Ziad Al Memish}

Research and Innovation Department, King Saud Medical City, Ministry of Health \& College of Medicine, Alfaisal University, Riyadh, Kingdom of Saudi Arabia \& Hubert Department of Global Health, Rollins School of Public Health, Emory University

\section{Megan Stanifer}

University Hospital Heidelberg and DKFZ https://orcid.org/0000-0002-5606-1297

\section{Steeve Boulant}

Department of Infectious Diseases, Heidelberg University Hospital

\section{Ronald Dijkman}

University of Bern https://orcid.org/0000-0003-0320-2743

\section{Anja Richter}

Institute of Virology, Charité-Universitätsmedizin

\section{Stefan Eggeling}

Vivantes Clinics Neukölln

\section{Andreas Hocke}

Charité - Universitätsmedizin Berlin

\section{Stefan Hippenstiel}


Charité - Universitätsmedizin Berlin https://orcid.org/0000-0002-5146-1064

\section{Volker Thiel}

Institute of Virology and Immunology, Bern, Switzerland https://orcid.org/0000-0002-5783-0887

\section{Stefan Pöhlmann}

German Primate Center - Leibniz Institute for Primate Research https://orcid.org/0000-0001-6086-9136

\section{Thorsten Wolff}

Robert Koch Institute https://orcid.org/0000-0001-7688-236X

\section{Marcel Müller}

Institute of Virology, Charité - Universitätsmedizin Berlin, corporatemember of Freie Universität Berlin, Humboldt-Universität zu Berlin, and Berlin Institute ofHealth https://orcid.org/0000-0003-2242-5117

\section{Christian Drosten ( $\square$ christian.drosten@charite.de)}

Charite - Universitätsmedizin Berlin https://orcid.org/0000-0001-7923-0519

\section{Article}

Keywords: Middle East respiratory syndrome coronavirus, MERS-CoV

Posted Date: July 15th, 2020

DOI: https://doi.org/10.21203/rs.3.rs-38496/v1

License: (c) (1) This work is licensed under a Creative Commons Attribution 4.0 International License. Read Full License

Version of Record: A version of this preprint was published at Nature Communications on September 7th, 2021. See the published version at https://doi.org/10.1038/s41467-021-25519-1. 


\section{Functional comparison of MERS-coronavirus lineages reveals}

2 increased zoonotic potential of the recombinant lineage 5

3

4 Simon Schroeder ${ }^{1}$, Christin Mache $^{2}$, Hannah Kleine-Weber ${ }^{3}$, Doreen Muth ${ }^{1}$, Victor M.

5 Corman $^{1,4}$, Diana Fatykhova ${ }^{5}$, Ziad A. Memish ${ }^{6,7,8}$, Megan Stanifer ${ }^{9}$, Steeve Boulant ${ }^{10,11}$,

6 Ronald Dijkman ${ }^{12}$, Anja Richter ${ }^{1}$, Stephan Eggeling ${ }^{13}$, Andreas Hocke ${ }^{5}$, Stefan Hippenstiel ${ }^{5}$,

7 Volker Thiel ${ }^{12}$, Stefan Pöhlmann ${ }^{3}$, Thorsten Wolff ${ }^{2}$, Marcel A. Müller ${ }^{1,4,14}$, Christian Drosten *1,4

8

$9{ }^{1}$ Institute of Virology, Charité-Universitätsmedizin Berlin, Berlin, Germany

102 Unit 17, Influenza and other Respiratory Viruses, Robert Koch Institut, Berlin, Germany

$11{ }^{3}$ Infection Biology Unit, German Primate Center - Leibniz Institute for Primate Research,

12 Göttingen, Germany

$13{ }^{4}$ German Centre for Infection Research (DZIF), Berlin, Germany

$14{ }^{5}$ Dept. of Infectious and Respiratory Diseases, Charité - Universitätsmedizin Berlin, Freie 15 Universität Berlin, Humboldt-Universität zu Berlin, and Berlin Institute of Health, Berlin, 16 Germany

$17{ }^{6}$ Research and Innovation Department, King Saud Medical City, Ministry of Health

$18{ }^{7}$ College of Medicine, Alfaisal University, Riyadh, Kingdom of Saudi Arabia

$19{ }^{8}$ Hubert Department of Global Health, Rollins School of Public Health, Emory University, 20 Atlanta, GA, USA

219 Department of Infectious Diseases, Molecular Virology, Heidelberg University Hospital, 22 Heidelberg, Germany

$23{ }^{10}$ Research Group "Cellular polarity and viral infection”, German Cancer Research Center 24 (DKFZ), Heidelberg, Germany

$25{ }^{11}$ Department of Infectious Diseases, Virology, Heidelberg University, Heidelberg, Germany

2612 Institute of Virology and Immunology, Bern \& Mittelhäusern, Department of Infectious

27 Diseases and Pathobiology, University of Bern, Bern, Switzerland

$28{ }^{13}$ Department of Thoracic Surgery, Vivantes Clinics Neukölln, Berlin, Germany

$29{ }^{14}$ Martsinovsky Institute of Medical Parasitology, Tropical and Vector Borne Diseases,

30 Sechenov University, Moscow, Russia

31 * corresponding author 


\section{Address for correspondence}

33 Professor Christian Drosten

34 Institute of Virology

35 Charité - Universitätsmedizin Berlin

36 Campus Charité Mitte

37 Chariteplatz 1

38 D-10117 Berlin

39 Germany

40 E-Mail: christian.drosten@charite.de 


\section{Abstract}

Middle East respiratory syndrome coronavirus (MERS-CoV) can cause severe pneumonia in humans. The virus is enzootic in dromedary camels across the Middle East and Africa. It is acquired through animal contact and undergoes limited onward transmission particularly in hospitals. Because of this initial potential for human-to-human transmission, we monitor the virus for phenotypic changes related to its pandemic potential. Potential phenotypic changes have been suspected since the year 2015, when a novel recombinant clade (MERS-CoV lineage 5) caused large nosocomial outbreaks in Saudi Arabia and South Korea that effectively swept other, hitherto co-circulating viral lineages. To this day, lineage 5 remains the only circulating MERS-CoV lineage on the Arabian Peninsula. In spite of available sequence data, no studies of viral phenotypes have been carried out to date. Here we performed a comprehensive in-vitro and ex-vivo comparison of live virus isolates sampled in Saudi Arabia immediately before and after the shift toward lineage 5 . We characterized seven isolates representing the recombination-parental lineage 3 , eight isolates representing parental lineage 4 , as well as eight isolates representing lineage 5. Replication of lineage 5 viruses is significantly increased over isolates from parental lineages in cell culture and ex-vivo lung models. Transcriptional profiling by real-time RT-PCR shows that several key immune genes (IFNb1, CCL5, IFNL1) are significantly less induced in lung cells infected with lineage 5 MERSCoV compared to parental strains. In interferon (IFN) receptor knock out cells, as well as under chemical inhibition of IFN signalling, the differences in replication level between lineage 5 and parental lineages are reduced, suggesting that phenotypic differences may be determined by IFN antagonism. Concordantly, lineage 5 shows increased resilience against IFN pretreatment of Calu-3 cells and maintains a 10-fold higher replication level under low and high concentrations of IFN. Reduced immune activation combined with enhanced virus replication and IFN resilience may explain the dominance of lineage 5 on the Arabian Peninsula. This phenotypic difference is highly relevant with regard to pandemic potential, and has remained undiscovered in spite of viral sequence surveillance. 


\section{Introduction}

The pandemic spread of severe acute respiratory coronavirus 2 (SARS-CoV-2) demonstrates the importance of monitoring emerging coronaviruses (CoV). Middle East respiratory syndrome coronavirus (MERS-CoV) is the causative agent of a novel form of severe viral pneumonia in humans [1]. MERS-CoV emerged in 2012 and the majority of currently more than 2,494 notified cases, including 858 deaths, occurred on the Arabian Peninsula. Travelassociated cases were diagnosed in 27 countries and sparked secondary case clusters in some. The largest MERS-CoV outbreak outside the Arabian Peninsula occurred in South Korea in 2015, involving 186 cases and 36 deaths (WHO, 2020).

MERS-CoV is acquired as a zoonotic infection from dromedary camels [2-5]. Spill-over from dromedaries to humans can lead to local outbreaks with limited human-to-human transmission $[6,7]$. Healthcare facilities can experience protracted outbreaks with severe infections in comorbid patients [8]. Behavioural factors like family patient care may accelerate outbreaks [9$11]$.

MERS-CoV phylogeny currently comprises three major clades, provisionally named clades $\mathrm{A}$, $B$ and $C$ [12-14]. Whereas clades $A$ and $C$ contain extinct strains and strains not circulating in the Arabian Peninsula, clade B strains currently infect humans and camels in this area. Clade $B$ is subdivided into six phylogenetic lineages. A presumed recombination between lineage 3 and 4 resulted in the formation of a circulating recombinant lineage (lineage 5, also termed NRC for novel recombinant clade) during or before the year 2014 in camels [14-17].

Surveillance of circulating viruses has been ineffective after 2015. Only based on more recent studies, it appears that lineage 5 has essentially replaced all other endemic strains since 2015 [18] (Figure 1). Because clade B lineages used to co-circulate widely before 2015, the novel dominance of a single viral lineage deserves clarification. Any change of phenotype might indicate alterations in the already existing potential for human-to-human transmission. Changes of phenotype in association with lineage 5 emergence have been suspected, but phenotypic studies of MERS-CoV strains are generally limited. Mutations in the spike protein positions I529T and D510G observed during the outbreak in Korea were suggested to have contributed to antibody evasion [19]. However, these polymorphisms evolved during and not prior to the Korean MERS-CoV outbreak and hence cannot explain the dominance of lineage 5 since 2015 [20]. Earlier studies looking into functional differences between MERS-CoV clades other than lineage 5 found little evidence for phenotypic differences [12, 21-23]. One complicating feature of MERS-CoV is that the infection phenotype as seen in humans is difficult to reflect in small animal models.

Here, in order to understand phenotypic traits of lineage 5 virus isolates, we used different cell, epithelial-, and ex-vivo human lung models to compare patient virus isolates belonging to lineage 5 as well as the parental lineages 3 and 4 . As MERS-CoV is known to act against the 
induction of cytokines and overcome the effects of antiviral genes [24-28], we investigated differences in immune gene activation and suppression of viral replication in response to interferon signalling. Lineage 5 viruses replicate more efficiently, show decreased antiviral IRF3- and NFkB-dependent signalling, and are less susceptible to interferon response.

\section{Results}

Phylogeny and recombination analysis of MERS-CoV isolates obtained from Saudi Arabian patients

To facilitate a phenotypic comparison of MERS-CoV strains that circulated before and after the 2014 recombination event, we generated 23 virus isolates from patient samples obtained during outbreaks in Saudi Arabia between 2014 and 2015 using a previously established MERS-CoV isolation protocol [23]. Early passage isolates were grown to high concentration, physically purified and characterized by ultradeep sequencing, real-time RT-PCR, and plaque quantification. No plaque purification was performed to avoid the introduction of selection bottlenecks. To further avoid cell culture-derived selection biases, all quantitative experiments were done on different representative isolates per viral clade with subsequent pooling of results.

123 For phylogenetic classification, we sequenced all isolates and inferred trees based on whole genome sequences (Supplementary Figure 1). All studied isolates cluster with either lineage 3 , lineage 4 or lineage 5 . As expected, lineage 5 branches from lineage 3 in a tree based on a full genome alignment $[14,15,17]$. Among the present isolates, seven belong to lineage 3 and originate from a MERS-CoV outbreak in Riyadh in 2014 [29]. Eight belong to lineage 4 and originate from a 2014 outbreak in Jeddah [22]. Another eight isolates belong to lineage 5 and stem from patients treated in Riyadh between September and November 2015. All show the typical pattern of topological incongruence when inferring trees from alignments that cover different genome portions relative to the two recombination breakpoints.

\section{Replication of MERS-CoV lineages in cell culture}

134 To compare viral replication we performed multicycle growth curves in Vero B4 monkey kidney cells that are widely used for diagnostic isolation of MERS-CoV. We observed no significant growth differences between isolates (Figure 2A). Because of the known deficiency of Vero cells in type 1 interferon induction, we also performed multicycle growth curve experiments in human lung- and colon carcinoma cell lines Calu-3 and Caco-2, respectively. These experiments detected significantly increased replication levels for all tested isolates pertaining to lineage 5. Growth was enhanced over that of the EMC reference strain, but also over that of all tested isolates pertaining to parental lineages 3 and 4 (Figure 2A-C). 
Two representative isolates per lineage were tested in these experiments initially. In order to minimize any influence of possible inter-isolate phenotypic variability, we used an extended range of virus isolates for the experiment in Calu-3 cells (Figure 2B). In each of four independent experiments, we used different sets of two viral isolates per lineage, resulting in each lineage to be represented by eight different viral isolates (refer to Supplementary Table 2 for virus isolates used in each experimental repetition). On broad average, all tested isolates pertaining to lineage 5 produced viral titers at least 5 -fold higher at 24 hpi than lineage 3 and lineage 4 isolates (Figure 2B and 2C). Within individual experiments, representatives of lineage 5 reached up to 10 -fold higher infectious titers than those of lineage 3 and 4 . The highly replicated experiment in Figure 2B yielded an average concentration of $1.23 \times 10 \mathrm{e} 5 \mathrm{PFU} / \mathrm{ml}$ for lineage 5 isolates compared to 2.2x10e4 PFU/ml for lineage 3 and $2.4 \times 10 \mathrm{e} 4 \mathrm{PFU} / \mathrm{ml}$ for lineage 4. All of these differences were highly significant. In addition the difference against MERS-CoV EMC was highly significant $(p<0.0006)$.

To better represent the conditions of viral replication at respiratory epithelia, we infected polarized Calu-3 cells grown under air-liquid interface conditions and found the same viral phenotypes (Figure 2D).

\section{Competitive replicative fitness}

Cell culture growth kinetics can suffer from systematic and random errors, parts of which can be controlled for by competitive replication studies in which viral isolates compete in one same culture dish. A given virus is likely to have superior relative fitness if it can become dominant in a virus population in spite of starting as a minority population in the initial virus seed dose used for infection [30]. To test this, Calu-3 cells were infected with a mixture of representative virus of lineage 5 and lineage 3 in two different ratios (1:1 and $9: 1$; lineage 3 : lineage 5). The initial seed dose in these cultures was set to 10,000 PFU, corresponding to an intermediate $\mathrm{MOI}=0.04$, which enables a short multicycle growth experiment while avoiding stochastic errors in the seed dose. As the total virus amplification from a single round of infection starting from this seed dose is too limited to detect robust differences in replicative fitness, four additional amplification cycles (passages) were performed. In preliminary experiments in the given cell culture format, we determined that the yield of infectious virus progeny when infecting Calu-3 cells at $\mathrm{MOI}=0.04$ will be $10 \mathrm{e} 4-10 \mathrm{e} 5 \mathrm{PFU} / \mathrm{ml}$ at $24 \mathrm{hpi}$. We therefore diluted passage supernatants to a new seed dose of ca. 5,000 PFU and repeated this process until completion of a total of five passages. We isolated viral RNA from the initial inoculum (p0) and from the supernatant after five passages (p5) and directly sequenced two different single nucleotide polymorphisms (SNP) sites that were each amplified from the virus population in three separate RT-PCR reactions to control for PCR-based artifacts. 
Based on Sanger sequencing we analyzed peak heights to quantify how much of each virus lineage was present at p0 and p5 (Figure 3A). In both analyzed positions we found the ratio of virus progeny at $\mathrm{p} 5$ to shift in favor of lineage 5 (Figure 3B).

\section{Analysis of stages of viral replication cycle}

183 Multiple factors might be responsible for enhanced MERS-CoV growth in cell cultures, 184 including improved virus attachment, entry, transcription, replication, infectious particle 185 production, or innate immune counteraction.

\section{Spike protein gene sequences}

We found no indications for differences in sialic acid-binding and receptor-binding properties in amino acid alignments of viral spike sequences (supplementary Figure 3 ). The $\mathrm{N}$ terminus mediates virus attachment via sialic acid domains. Its sequence is identical in all four lineages and in silico analysis by NetNGlyc and NetOGlyc showed no differences in glycosylation patterns. The receptor-binding domain (RBD) is responsible for binding to the entry receptor, DPP4. The four lineages further share the same amino acid sequence in the RBD, with the exception of lineage 3 isolates showing one polymorphism (L411F). However, previous studies of this polymorphism demonstrated unaltered binding affinity and entry efficiency [19].

\section{Cell entry capacity and receptor binding}

To explore cell entry capacity and receptor binding, we produced expression vectors carrying the spike proteins of lineage 3 , lineage 4 and lineage 5 strains and incorporated these into vesicular stomatitis virus (VSV)-based pseudotypes [19]. We tested entry capacity in cell lines expressing high and low amounts of TMRPSS2 (high: Calu-3 and Caco-2, Figure 4A and 4B; low: Huh7, Figure 4C) to account for entry capacity with protease-primed and non-primed spike protein [31]. No differences were observed between pseudotypes.

We also expressed the lineage-specific spike proteins in 293T cells and measured the binding capacity to the MERS-CoV entry receptor, DPP4, by flow cytometry as described in [19]. Again, we found no significant differences between spike proteins of each lineage (Figure 4D).

\section{Full virus entry studies}

209 To compare the entire entry process, we performed single cycle $(\mathrm{MOI}=1)$ infection assays in 210 Calu-3 lung cells using full virus isolates. We infected all cell cultures at $4^{\circ} \mathrm{C}$ to ensure that cell 211 entry is initiated simultaneously by temperature shift to $37^{\circ} \mathrm{C}$. Onset of sgRNA N transcription, 212 as observable before the onset of genome replication, was taken as an early indicator of the 213 conclusion of the entry process. As a control, we simultaneously blocked entry of MERS-CoV 214 on several levels using inhibitors of clathrin-mediated endocytosis (classical entry pathway), 
215 host membrane serine proteases (alternative direct entry pathway), as well as endosomal

216 proteases (downstream endosome fusion). We detected no significant differences in genomic

217 and subgenomic RNA quantities among all virus lineages 1 and 4 hours post binding (Figure

218 5). Chemical blocking of virus entry revealed that all four lineages had entered into the stage

219 of early subgenomic RNA transcription by 4 hours post adsorption, without discernible

220 differences in transcription levels.

\section{Virus neutralisation by anti-MERS-CoV-positive human and camel sera}

223 Because neutralizing antibodies may act via proteins or domains other than the spike $\mathrm{N}$ 224 terminus and RBD, we tested serum neutralization based on a highly sensitive plaque 225 reduction neutralization assay on live viruses. All virus isolates are neutralized with equal 226 efficiency by human $(\mathrm{N}=2)$ and camel $(\mathrm{N}=3)$ sera as summarized in Supplementary Table 1 227 [11].

\section{Early viral replication}

230 Whereas above experiments used synchronized infection at high $\mathrm{MOI}$, we also compared multi-cycle, low MOI replication experiments in which the early phase of replication is less masked by input viral RNA and the early onset of transcription may not be discernible from genome replication [24]. Calu-3 and VeroB4 cells were inoculated at $4^{\circ} \mathrm{C}$ to ensure that virus particles attached with equal efficiency to the cellular receptors and the cell entry process started simultaneously. We quantified subgenomic mRNA and genomic RNA transcription and measured PFU/ml in supernatant to analyze putative differences in virus particle formation and egress (Figure 6). There was little difference between $\mathrm{N}$ and genomic transcripts. Virus infections in Calu-3 cells showed an earlier onset of replication than in Vero cells, which may be attributable to the availability of TMPRSS2 providing an additional entry pathway in Calu-3 cells [32]. Already from the beginning of detectable replication, lineage 5 viruses show a higher level of RNA transcription than the parental lineages 3 and 4. Enhanced infectious virus production of lineage 5 was only seen in Calu-3- but not in type I interferon- and TMPRSS2deficient VeroB4 cells, which corresponds to our previous observations (Figure 2). As experiments up to this point have largely excluded a role of TMPRSS2-dependent entry, we suspected the phenotypic difference to be linked to cell-intrinsic innate immunity and in particular the type I interferon system.

\section{Differences in cytokine induction}

249 To explore if higher replication is reflected in higher cytokine induction, we infected Calu-3 cells 250 with two viruses of each phylogenetic lineage in a single-cycle infection for 12 hours and analysed the mRNA expression levels of a set of immune-related genes. We chose IRF3- 
regulated genes IFNb1 and IFNL1, NFkB-regulated genes CCL5 and TNFa, as well as IFNstimulated gene Mx1. To enable single cycle virus infection, cells were infected at $\mathrm{MOI}=2$ (Figure 7A). Also under these conditions, the two lineage 5 isolates used in the experiment replicated to a higher level than the members of lineage 3 and 4 (two strains each). To our surprise, lineage 5 induced significantly lower levels of IFN and CCL5 mRNA compared to isolates pertaining to lineage 3 and 4 (Figure 7B). IFNb1 and IFNL1 mRNA expression levels were reduced in cells infected with lineage 5. Immune gene mRNA induction in general seemed to be highest with lineage 3 strains.

260

\section{IFN sensitivity}

262

Previous studies have shown that MERS-CoV EMC is highly sensitive towards type I IFN pre263 treatment [52]. To analyze IFN sensitivity among the different MERS-CoV lineages, we compared virus replication in Calu- 3 cells pre-treated with type I interferon for 16 hours. We chose IFN concentrations that induce mainly an upregulation of cellular helicases (2.5 units IFN), as well as IFN concentrations high enough to induce a full antiviral state ( 25 units) in treated Calu-3 cells.

As expected [33], replication of the reference strain EMC was already suppressed by low level IFN pretreatment. Lineage 3 strains showed similarly high sensitivity, while lineage 4 and lineage 5 strains were not suppressed by 2.5 units of IFN (Figure 8). 25 units of IFN markedly reduced virus replication of all lineages. The reduction of replication caused by this dose was lower for lineage 5 than strains pertaining to lineage 3 and 4 (6.5-fold versus 23 and 18-fold, respectively).

274

275

\section{Influence of IFN action via the JAK/STAT signalling pathway}

277 Because above observations might be attributable to direct IFN action but could also be 278 attributable to the observed lower level of cytokine induction by lineage 5 strains, we more specifically studied the influence of JAK/STAT - mediated IFN signalling. We infected Calu-3 cells in the presence of the JAK/STAT inhibitor Ruxolitinib (Invivogen) (Figure 9A), and also compared virus replication in MERS-CoV susceptible cells carrying an IFN-alpha and -lambda receptor double knock-out (T84 cells, Figure 9B). Whereas control experiments confirmed the higher replication levels of lineage 5 , both approaches to IFN receptor inactivation caused lineage 5 strains to only lose a part of their replicative prominence. Overall, lineage 5 strains retained a significantly higher replication level than strains pertaining to lineage 3 and 4 , suggesting that IFN action alone does not explain the observed differences. 


\section{Replication phenotype in models of the human respiratory tract}

289 To more closely reflect virus replication in the respiratory tract [41], we infected differentiated 290 human airway epithelia (HAEs) with two representative isolates from each phylogenetic 291 lineage. According to our previous observations [45] we sampled supernatants exclusively 292 from the apical site of differentiated HAE and quantified virus progeny every 24 hours for four 293 subsequent days. Lineage 5 isolates reached average titres up to 15 -fold higher compared to 294 lineage 3, lineage 4 and the EMC reference strain, with significant differences at 24 and 48 hpi 295 (Figure 10A).

296 To provide a model of infection that most closely resembles infection of the human lung, we 297 performed ex-vivo infections in human lung explants, derived from patients that have 298 undergone lung resection. We used one representative virus isolate per phylogenetic lineage 299 to infect lung explants derived from three different donors (Figure 10B). Supernatants of 300 infected lung explants were harvested at 16, 24, 48 and 72 hpi. Similar to HAEs, lineage 5 301 reached titers up to 5 -fold higher compared to lineage 3 , lineage 4 and the EMC reference 302 strain, with significant differences at 24 and $48 \mathrm{hpi}$ (Figure 10B). Higher ex vivo replication of 303 lineage 5 viruses by confirmed by immunofluorescent analysis of infected lung explants 304 (Figure 10C). We quantified the area staining positive for MERS-CoV infection in three lung 305 306 explants, derived from three donors, and found averages of infected areas to be highest in samples infected with MERS-CoV lineage 5, at 24 hpi (Figure 10D).

\section{Discussion}

308 The present study demonstrates that a newly emerged genetic lineage of MERS-CoV has 309 higher replicative fitness and causes a lower level of cytokine induction than previouslycirculating strains. An example of recombination between coronavirus lineages giving rise to a more pathogenic strain has already been provided by the cases of feline coronaviruses and IBV [34, 35]. To our knowledge, the present study is the first report of a recombinant coronavirus lineage with change of phenotype in humans. Its occurrence in MERS-CoV is particularly relevant from a public health perspective. Since 2015, lineage 5 was highly prevalent in sampled dromedary camels and humans in Saudi Arabia, and caused a major outbreak in South-Korea [14-17]. This upsurge triggered speculations as to the transmissibility of the novel virus variant. Nevertheless, the phenotype of these viruses, as opposed to viruses circulating earlier, has never been studied. The present study provides a first comprehensive phenotypical assessment of lineage 5 isolates, showing changes that likely correlate with transmissibility and virulence. Notably, a higher relative fitness and increased replication level may translate into higher excreted virus doses during infection. If higher doses are transmitted on average, this will inevitably increase the viral capability to adapt to humans [30, 36]. In 
addition, reduced cytokine induction is often considered to be an indicator of increased

324 virulence.

325 The functional diversity of circulating viruses in KSA is somewhat surprising given that earlier 326 studies found little indication for phenotypic differences between viral lineages [12, 17, 22, 37]. 327 A study of African viruses found functional diversity [12] that could be attributed to deletions in 328 ORF4b encoding a suppressor of RNAse $L$ that acts via an active phosphodiesterase function $329[25,38]$. However, the present viruses have a full gene repertoire and thus provide little angle 330 to link changes of phenotype to any defined gene target. The recombination event that created 331 lineage 5 may itself be causal if individually neutral mutations from donor lineages were 332 shuffled together to increase fitness though epistasis. Alternatively, fitness-increasing 333 mutations could have occurred after the recombination event. There is some evidence from 334 our data that members of the parental lineage 4 show an attenuated cytokine-based immune response, but these viruses do not show increased fitness overall. We can conclude from our experiments that the increased fitness of members of lineage 5 does not result from changes affecting viral entry and is unlikely to be based on an altered interference with IFN receptordependent signaling. Most likely, the differences in this part are caused secondarily due to clear differences in either infection sensing or downstream elements of cytokine induction cascades. The further search for mechanisms to explain viral phenotype and host response will have to involve comprehensive studies such as shuffling of gene portions based on reverse genetics. Identifying causal mutations will help to understand whether and how cooperating mutations may have been assembled into one genome following recombination, and how the recombinant virus phenotype was selected for. Mapping those causal mutations to functional domains within the viral genome might even be used to assess the pathogenicity of newly emerging MERS-CoV.

347 It is a limitation of the present study that deeper mechanistic investigation cannot be provided.

348 We note that the observed differences in virus-host interaction may originate outside the direct 349 infection sensing and cytokine induction mechanisms, so that changes in cytokine induction as well as the partial differences in IFN sensitivity might be collateral effects. In addition, it should be clearly understood that the observed phenotype of lineage 5 does not implicate selection in humans. Analyses of viral populations have led to the conclusion that human-tohuman transmission does not play a relevant role in MERS-CoV evolutionary dynamics, and selection therefore will have taken place in the animal reservoir [39]. However, the reduced induction of cytokine expression is compatible with selection in camels as immune sensors and appending signal transduction cascades triggering cytokine induction are conserved among mammals. Selection for virulence in camels may involve a collateral benefit for the virus once transmitted to humans. 


\section{Materials and Methods}

\section{Viruses}

All virus stocks have been primarily isolated from respiratory samples, obtained from Saudi Arabian patients diagnosed with MERS between March 2014 and November 2015. Patient material was obtained from collaboration partners of different regional laboratories or hospitals, all in the KSA. Highly q-RT-PCR positive samples were selected for primary virus isolation as described in [40, 42]. Successful primary isolates were used for high titer stock production in VeroB4 cells and were concentrated using Vivaspin columns (100,000 MWCO PES membrane; Sartorius, Germany) upon reaching maximal cytopathic effect after daily monitoring. All stocks were quantified using plaque titration as described below.

\section{Virus sequence analysis}

Viral RNA of 22 passage two virus stocks was extracted using the Machinery and Nagel viral RNA kit. Viral RNA was subjected to cDNA synthesis using the SuperScript ${ }^{\circledR}$ One-Cycle cDNA Kit (Invitrogen) according to the manufacturer's protocol. cDNA was fragmented and supplemented with adapter sequences using the Nextera® XT DNA Library Prep (Illumina), subsequently processed with the MiSeq Reagent Kit v3 (Illumina) and subjected to NGS on a MiSeq ${ }^{\mathrm{TM}}$ System (Illumina), all according to the manufacturer's protocol. Using the geneious software, version 9.1.8, contigs were assembled by mapping reads to the EMC genome (genbank ref.: JX869059). Assembled full genomes have been deposited to NCBI GenBank under the accession numbers MN481964 to MN481978.

\section{Phylogeny and recombination analysis}

383 Phylogenies were estimated by Maximum-likelihood approach, implemented in RaxML, version 8.0.0, with a general time reversible (GTR) model of nucleotide substitution with estimated gamma distributed rate variation and a proportion of invariant sites. 10,000 bootstrap replicates were run. Input alignments of whole genome and subgenomic sequences comprising the outer and inner non-recombinant segments were aligned by implementing ClustalW in the geneious software, version 9.1.8.

For detailed recombination analysis, we performed a BootScan analysis with 500 bootstraps in SimPlot, version 3.5.1., with a sliding window of 5000 bp and 200 bp steps.

\section{General cell culture procedures}

393 All cells were maintained in Dulbecco's Modified Eagles Medium (DMEM) at $37^{\circ} \mathrm{C}, 5 \% \mathrm{CO}_{2}$ as described earlier [40]. For virus titration VeroB4 cells were used. For infection studies Vero B4 (DSMZ-AC33), Calu-3 (ATCC HTB-55) and Caco-2 (ATCC HTB-37), T84 (ATCC-CCL-248) and T84 IFN alpha and lambda receptor double knockout cells were used. For polarization, 
Calu-3 cells were seeded on transwell inserts (Greiner Bio, pore size $0.4 \mu \mathrm{m}$ ) and initially cultivated under liquid-liquid conditions for 3 days. Subsequently cells were transferred to the air-liquid interface (ALI) condition with basolateral medium exchange every 2 days for 14 days.

400 Prior to infection, complete polarization was confirmed by transepithelial electrical resistance measurement. For VSVpp transduction studies, 293T, Caco-2, Calu-3 and Huh-7 cells were

402

403

404

405

406

407

408

409

410

411

412

413

414 used and maintained as described above. Human airway epithelia (HAE) and human lung explants were generated and maintained as described in [40] and [41], respectively.

\section{Lung explants infection, culture procedure and immunofluorescent analysis}

Lung explants were obtained from patients undergoing lung resection. Written informed consent was obtained from all patients and the study was approved by the ethic committee at Charité clinic (project EA2/079/13). For each experiment tumor-free human lung tissue was cut into small pieces (weight approx. 0.1 - $0.2 \mathrm{mg}$ per piece) and incubated in RPMI 1640 medium at $37^{\circ} \mathrm{C}$ with $5 \% \mathrm{CO} 2$. After overnight incubation lung organ cultures were inoculated with $1 \times 10 \mathrm{e} 5 \mathrm{PFU}$ of virus for $1.5 \mathrm{~h}$ under shaking conditions followed by one washing step with phosphate-buffered saline (PBS) to remove excess virus. Lung tissue was incubated for up to 72 hpi in RPMI 1640 medium containing 10\% fetal calf serum and 2 mM L-glutamine. For growth curve analysis supernatants of infected lung tissue were harvested at 0, 16, 24, 48 and $72 \mathrm{hpi}$ and supernatants were titrated on Vero E6 cells by standard plaque titration assay.

For immunofluorescent analysis, processing of human lung tissues was performed as described in [42]. In brief, MERS-CoV infected tissue samples were fixed in $3 \%$ paraformaldehyde twice for $24 \mathrm{~h}$. Samples were subjected to anti-MERS-CoV nucleocapsid primary antibody (Sino Biological, China) detection overnight at $4{ }^{\circ} \mathrm{C}$ and labelled with a secondary antibody Alexa Fluor 488 (Invitrogen, Darmstadt, Germany). Nucleus counterstaining was performed with DAPI (Sigma Aldrich, Germany). Immunofluorescence spectral confocal microscopy was conducted using a LSM 780 [(objectives: Plan Apochromat 63x/1.40 oil DIC M27), Carl-Zeiss, Jena, Germany]. Based on a spectral image lambda stack, linear unmixing of tissue autofluorescence and overlapping spectra of fluorochromes were performed using ZEN 2012 software (Carl-Zeiss, Jena, Germany). Each image showns a maximum fluorescence intensity projection of a z-stack representing the entire $10 \mu \mathrm{m}$ section.

To reveal lung and cell morphology, images were combined with Differential Interference Contrast (DIC). All image sets were acquired using equal configurations. Images were processed using ZEN 2012 and ImageJ software (http://imagej.nih.gov/ij/). Quantification of Alexa Fluor 488 signal intensity was performed using ZEN 3.1 (blue edition; Carl-Zeiss, Jena, Germany). Briefly, stained human lung slides were scanned using Axio scan.Z1 (Carl-Zeiss, Jena, Germany) followed by digital image segmentation of positive-infected areas in $\mu m^{2}$ and 
433 normalization to the total area of the lung slices, measured by autofluorescence of the lung

434 tissue.

435

\section{Virus Infections}

437 VeroB4 and Caco- 2 cells were seeded at $1.5 \times 10$ e 5 cells/well, Calu- 3 cells at $3 \times 10$ e 5 cells/well and T84 cells at $2 \times 10$ e 5 cells/well, all in composite DMEM in 24-well plates 16 hours prior to infection. Cultured cells (VeroB4, Calu-3, Caco-2, T84 cells) were infected with passage two MERS-CoV stocks diluted to the desired $\mathrm{MOI}$ in OptiPro for one hour at $37^{\circ} \mathrm{C}$. HAEs were infected with 4000 plaque-forming units (PFU) in Hanks Balanced Salt Solution (HBSS) for 1.5 hours. Subsequently, cells were washed three times with PBS or HBSS for HAEs, respectively, and further incubated. At 24 and 48 hours, respectively 24, 48, 72 and 96 hours for HAEs, supernatants were harvested and viral loads were quantified by plaque titration on VeroB4 cells as described below. For IFN priming experiments, Calu-3 and VeroB4 cells were treated 24 hours post seeding with either $0,2.5$ or 25 units pan-species type I IFN for 16 hours prior to infection.

\section{Synchronized infections and virus host cell entry blocking}

450 For virus cell entry experiments, Calu-3 cells were seeded 16 hours before infection in a 24well format at a seeding density of $3 \times 10 \mathrm{e} 5$ cells/well. 1 hour prior to virus infection, cells were washed twice with PBS and treated with composite DMEM without FCS, containing only DMSO (no inhibitors) or DMEM without FCS, containing a triple combination of previously described

454 CoV entry inhibitors resolved in DMSO (inhibitor samples). Cathepsin L inhibitor MDL28170 and Pitstop II (both Sigma-Aldrich) were used at a final concentration of $25 \mu \mathrm{M}$, Camostat mesylate (Sigma-Aldrich) was used at a final concentration of $100 \mu \mathrm{M}$. Calu-3 cells infection was performed on ice and the cells were immediately put on $4^{\circ} \mathrm{C}$ for 1 hour after pipetting virus dilutions onto the cells to allow for virus attachment for the cells without receptor mediated virus entry. Cells were subsequently washed five times with PBS to remove the majority of unbound virus particles and either immediately lysed (Ohpi samples), or incubated with composite DMEM, with or without the inhibitor cocktail, for 1 or 4 more hours post infection (1 hpi and $4 \mathrm{hpi}$ samples), prior to medium removal and cell lysis. RNA isolation of cell lysates and quantitative real-time PCR was performed as mentioned below.

\section{Plaque titration and plaque reduction neutralization test}

466 VeroB4 cells were seeded at $1.5 \times 10^{\wedge} 5$ cells/well in 24-well plates 16 hours prior to titration, as 467 described in [56]. A 1:2 mixture of Avicel (FMC BioPolymer, Brussels, Belgium) and double concentrated DMEM was used as overlay. Four days post infection, cells were washed, fixed 
neutralization tests (PRNT) was performed as described in [3]. In brief, 25 PFU of each isolate were incubated with serial diluted serum for $1 \mathrm{~h}$ at $37^{\circ} \mathrm{C}$, prior to plaque titration. The following five sera were used for neutralization: Munich-1 (MERS patient, Germany 2014), SA278 (MERS patient, KSA 2014), Dubai-S1 (camel, UAE 2014), Kenia-ILRI (camel, Kenya 2017) and Pakistan-493 (camel, Pakistan 2017).

\section{Cloning of lineage specific Spike genes and generation of vesicular stomatitis virus} pseudotype particles (VSVpp) and transduction of target cells

Previously cloned pCG1 vector carrying the EMC spike gene was subjected to mutagenesis PCR to accommodate the three amino acid substitutions specific for lineage 3 , lineage 4 and lineage 5 (supplementary Figure 3). In a first round of mutagenesis PCR, the Q1020R mutation was introduced (all lineages). The obtained plasmid was then subjected to a second round of mutagenesis PCR, introducing either L411F (lineage 3) or Q833R (lineage 5). 5'phosporylated primers were used for mutagenesis PCR as follows: Q1020R mutagenesis: forward: 5'TAATGAAGCTTTTCGGAAGGTTCAGGATGC-3';

reverse:

$5^{\prime}-$ GTTGTAGTGAAGCCTGTTTGCATAGCTCCC-3'. L411F mutagenesis: forward: 5'CCAATTGCAATTATAATTTTACCAAATTGCTTTCACTTTTTTCTGT-3'; reverse: 5'TAAAAACCAAACGCTTGAAATTATAAACCTGAGGAGGT-3’. Q833R mutagenesis: forward: $5^{\prime}-$ CAAAACTGGCCATACTCGCGCAGTAATTGC-3'), with the underlined nucleotides being the substitutional sequences.

Obtained pCG1 plasmids were used for the production of rhabdoviral spike proteins pseudotyped vectors, generated according to a previously published protocol [19]. Briefly, 293T cells transfected with pCG1 vectors, carrying EMC-Spike, MERS-S Q1020R, MERS-S Q1020R/L411F, MERS-S Q1020R/Q833R, VSV-G (positive control) or empty expression plasmid (negative control) were inoculated with replication-deficient, VSV-Gtranscomplemented vesicular stomatitis virus (VSV) in which the VSV-G gene was replaced by eGFP and firefly luciferase, VSV* $\Delta G$ (VSV-G) [43] that was kindly provided by Gert Zimmer. After $1 \mathrm{~h}$ incubation at $37^{\circ} \mathrm{C}$, the supernatant was removed, the cells washed with PBS and fresh culture medium was added. In case of spike protein-expressing cells or cells transfected with empty expression vector, the medium was supplemented with the VSV-G-neutralizing antibody 11 (produced from CRL-2700 cell line, ATCC).

For transduction of target cells in 96-well format, the culture medium was removed and cells were inoculated with $100 \mu \mathrm{l} \mathrm{VSVpp.} \mathrm{At} 18 \mathrm{~h}$ postinoculation cells were lysed for $30 \mathrm{~min}$ at RT in $50 \mu \mathrm{l}$ Luciferase Cell Culture Lysis Reagent (Promega) were added. Lysates were transferred into white, opaque-walled 96-well plates and activity of virus-encoded luciferase 
was measured using the Luciferase Assay System substrate (Promega) and the Hidex Sense plate reader (Hidex).

\section{Quantification of MERS-S binding to DPP4 by flow cytometry:}

Analysis of S protein binding to DPP4 was analyzed by flow cytometry employing a previously published protocol [19]. In brief, 293T cells were untransfected or transfected with the overexpression vector pCG1 either empty or carrying MERS-CoV Spike protein of clade A (EMC), EMC 1529T, lineage 3 (L411F/Q1020R), lineage 4 (Q833R/Q1020R), or lineage 5 (Q1020R), as well as empty vector for negative control. At $48 \mathrm{~h}$ post transfection, cells were resuspended in PBS, centrifuged ( 5 min at $600 \times \mathrm{g}$ at $4{ }^{\circ} \mathrm{C}$ ) and resuspended in PBS containing $1 \%$ bovine serum albumin ( $1 \%$ BSA/PBS) for washing. The cells were centrifuged again and incubated in $1 \%$ BSA/PBS containing soluble DPP4 equipped with a C-terminal human Fctag (solDPP4-Fc,1:50, 1:200 and 1:1000; ACROBiosystems) at $4{ }^{\circ} \mathrm{C}$ in an overhead shaker for $1 \mathrm{~h}$. Afterwards, the cells were pelleted and incubated in $1 \%$ BSA/PBS containing an AlexaFlour488-conjugated anti-human antibody (1:500; ThermoFisher Scientific), for $1 \mathrm{~h}$ at 4 ${ }^{\circ} \mathrm{C}$ in an overhead shaker. Subsequently the cells were pelleted, washed with $1 \%$ BSA/PBS and fixed in $4 \%$ paraformaldehyde solution for $20 \mathrm{~min}$ at room temperature. Prior to analysis via flow cytometry, the cells were pelleted again and washed with $1 \%$ BSA/PBS. Flow cytometry was conducted on an LSR II Flow Cytometer. Data was further processed in the FCS Express 4 Flow research software (De Novo software).

\section{Direct competition assay:}

528 Virus stocks of one lineage 5 isolate (Riyadh-1764-2015) and one lineage 3 isolate (Riyadh1147-2014) were mixed at two ratios (1:1 and 1:9 lineage 5:lineage 3 ) and used to infect Calu3 cells in duplicates at an moi 0.04 (corresponding to 10,000 total PFU). As a control, single infection with only 10,000 PFU of the lineage 5 or the lineage 3 isolate was performed. At $24 \mathrm{hpi}, 100 \mu \mathrm{l}$ of the cell culture supernatants of infected cells were used to inoculate new Calu3 cells for five subsequent passages. Viral RNA was isolated from each inoculum (passage 0/p0) and after five passages (passage 5/p5) using the Machinery and Nagel vRNA kit and three short PCR amplicons were reverse transcribed (SSIII/Taq one-step; Thermo Scientific) using specific primers (amplicon 1, 300bp: forward 5'-TACCTGGTTGAGAGGCTCAT-3'; reverse 5'-CTTAAGCAGATTCTGGGCATATT-3'; amplicon 2, 541bp: forward 5'TGAGTGTGGAAGTTGTGGTAAT-3”; reverse 5'-ACCTTTGAGAAGCTGGCGTATT-3'; amplicon 3, 560bp: forward 5'-TCGAGCCGCATAAGGTTCAT-3'; reverse 5'540 GCTGAGCTGCGTCCTGTTT-3'). The obtained PCR amplicons was sent for Sanger 541 sequencing. For peak height analysis, the web based Chromat Quanitator (Mullins lab, 542 University of Washington) was used and quantified sanger sequencing peak heights were averaged for all duplicates and calculated as a percentile of the total height. 
545 For quantitative real time PCR analysis, total RNA of MERS-CoV infected Calu-3 cells was 546 harvested at 12hpi using the Machinery and Nagel total RNA kit, following the manufacturer's 547 instructions. A two step PCR protocol was used for quantification of host transcripts. First, 548 cDNA was prepared from equal molar viral RNA using the iScript ${ }^{\mathrm{TM}}$ cDNA synthesis kit (Bio549 Rad), according to the manufacturer's protocol. Second, cDNA was amplified and quantified 550 using Taq-polymerase (Invitrogen) and a set of real time primer and probes for each transcript 551 under investigation (sequences available on request). For sgRNA N and ORF1a RNA analysis, 552 cells were harvested at 0, 2, 4, 6, 8, 10, 12 and 24hpi and total RNA was isolated and subjected 553 to quantitative real-time PCR using the one-step SuperScript III kit (Invitrogen) with primers 554 published in [44] and [45].

555

\section{Stastical Analysis}

557 Student's t-test or Mann-Whitney test were applied for comparisons of paired means. Analysis 558 of variance (ANOVA) with Bonferroni's post hoc test was used for group-wise comparisons. 559 Significance testing results are indicated by asterisks in the graphs. All statistical tests were 560 two-tailed and $p<0.05$ was considered statistically significant.

\section{Author contributions}

562 S.S., C.D. and M.A.M, conceived and designed the experiments. S.S., C.M., H.K.W, D.F. and 563 A.R. performed experiments. S.S., D.M. and V.C.M. performed virus isolations from clinical 564 samples. Z.A.M. provided clinical samples. M.L.S., S.B., R.D., S.E., A.C.H., S. H. and V.T. 565 provided essential materials. S.S., C.D. and M.A.M. wrote the manuscript. S.S. prepared all 566 figures.

\section{Acknowledgments}

568 The work was supported by the Bundesministerium für Bildung und Forschung under Grant 569 01KI1723A (RAPID), as well as the European Union via Project ReCoVer. S.B. is supported 570 by the Heisenberg program (project number 415089553 ) and M.L.S is supported by the DFG 571 (project number 41607209). A.C.H and S.H. were additionally supported by DFG (SFB-TR 84) 572 and Charite 3R; additionally A.C.H by Charité-Zeiss MultiDim. We thank Abdulla M. Assiri, 573 Public Health Directorate, Ministry of Health, Riyadh, for provision of additional clinical 574 samples.

\section{Competing interests}

576 The authors declare no competing interests. 


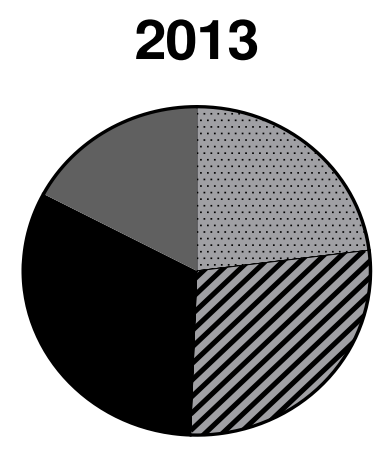

$\mathrm{n}=\mathbf{8 7}$

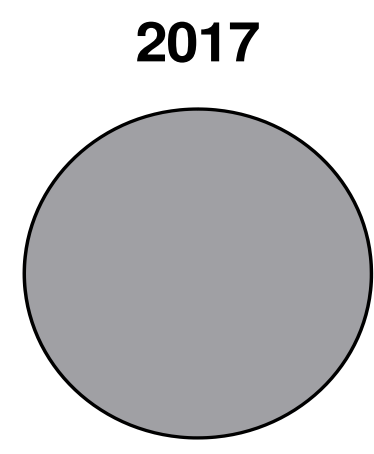

$\mathbf{n}=\mathbf{2 7}$

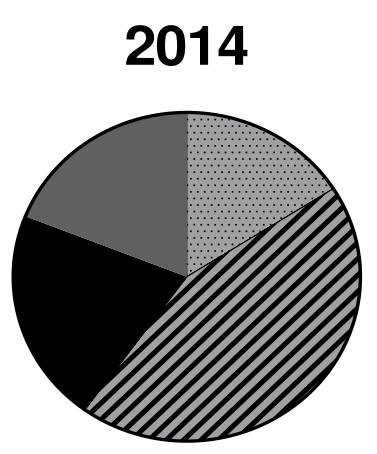

$n=100$

2018

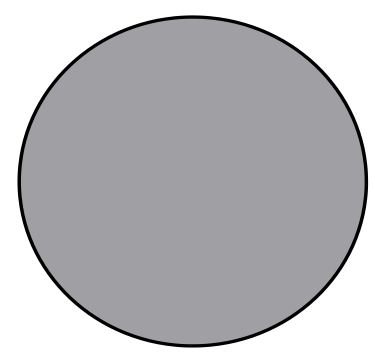

$n=13$

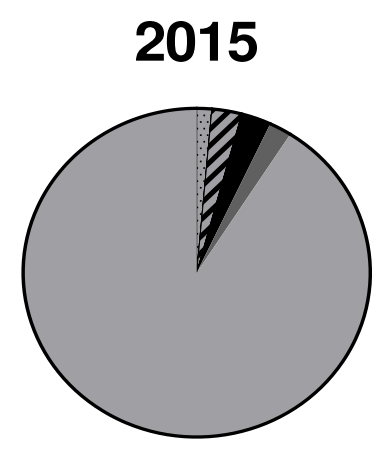

$\mathbf{n}=\mathbf{2 8 7}$

2019

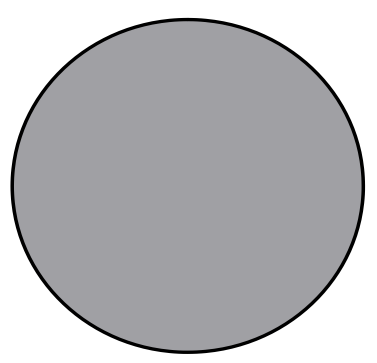

$n=29$
578

579

580 Figure 1: MERS-CoV sequences uploaded to NCBI GenBank between 2013 and 2020. All

581 available GenBank entries for MERS-CoV with a sequence length $\geq 5000$ nucleotides were 582 included $(n=561)$. The collection date provided in GenBank was used for assigning a year to 583 each sequence. lineage 1

$\square$ lineage 2

a lineage 3

$\square$ lineage 5

$\square$ lineage 5/NRC

\section{Figures with legends:}



A VeroB4
B Calu-3
C Caco-2
$24 \mathrm{hpi}$
D polarized Calu-3
$24 \mathrm{hpi}$
24 hpi
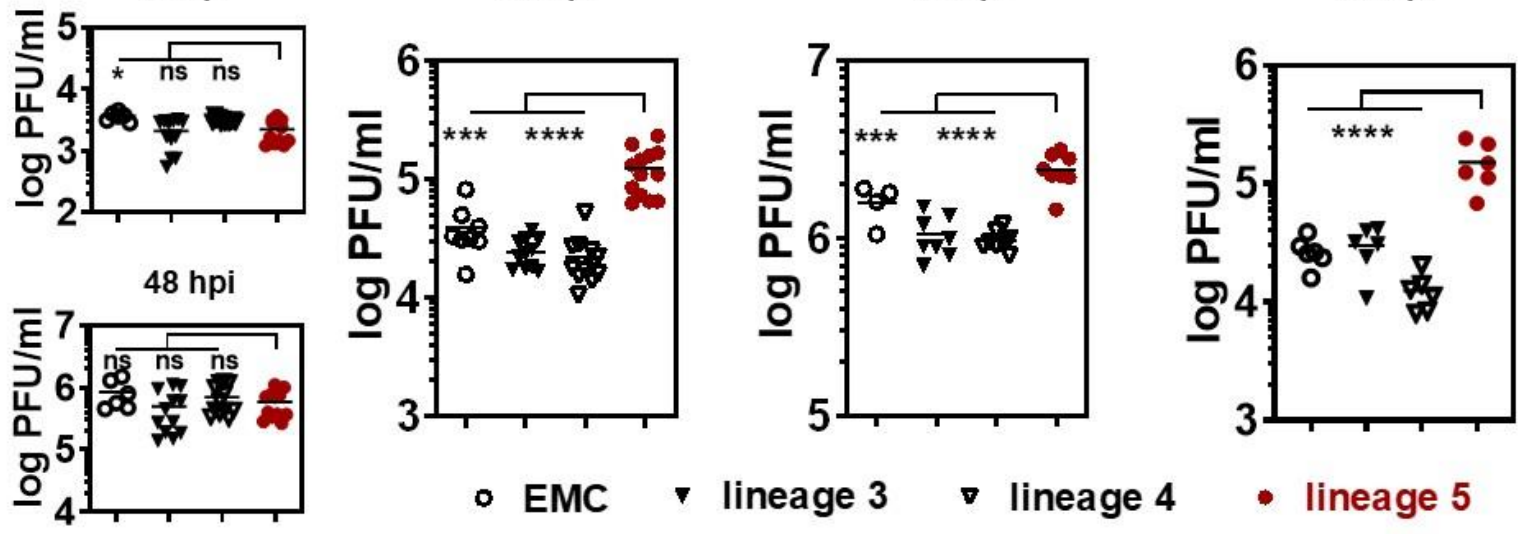

- EMC $\vee$ lineage $3 \nabla$ lineage 4

- lineage 5

584

585

586

587

588

589

590

591

592

593 $594 \quad 0.0001)$

Figure 2. Enhanced replication of lineage 5 virus isolates on permanent and primary cells. Cells were infected at $\mathrm{MOI}=0.002$ and virus progeny in supernatant quantified by plaque assay in cell culture supernatants. A) VeroB4 cells, 24 and 48 hpi; B) Calu-3 lung cells, 24 hpi C) Caco-2 colon cells, $24 \mathrm{hpi}$; D) Calu-3 lung cells grown as air-liquid interface culture, $24 \mathrm{hpi}$.

90 (polarized Calu-3), or $n=2$ independent experiments (Caco-2, VeroB4 and polarized Calu-3 591 cells) with $n=3$ virus isolates per lineage. Statistical significance in difference of PFU/ml 992 between lineage 5 and other lineages was determined by Krustall-Wallis test in $A)\left({ }^{*} P>0.0332\right.$,

ns $P<0.05)$, and by two-tailed t-test in B), C) and D) $\left({ }^{* *} P<0.0021\right.$; ${ }^{* *} P<0.0002$; ${ }^{* * \star} P<$ 
A Position 1 (nt 668)

Single infection

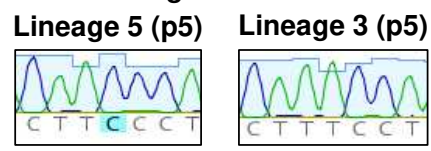

Lineage 5 + Lineage 3 (1:1)

(p0)
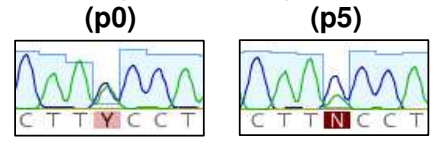

Lineage 5 + Lineage 3 (1:9)

(p0)

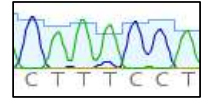
(p5)

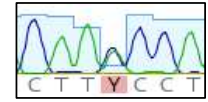

B

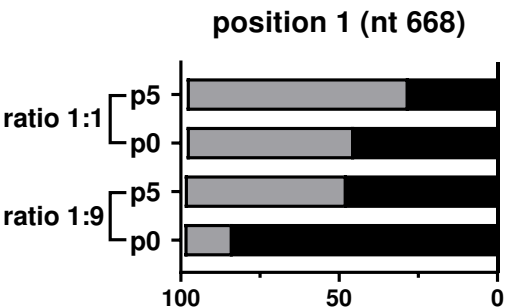

percent of lineage 5 and lineage 3 determined by sanger sequencing
Position 2 (nt 1514)

Single infection

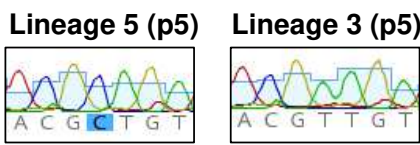

Lineage 5 + Lineage 3 (1:1) (p0)
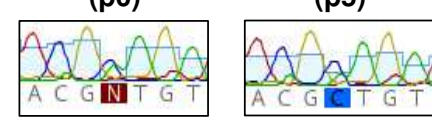

Lineage 5 + Lineage 3 (1:9) (p0)

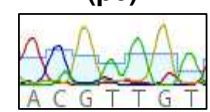

(p5)

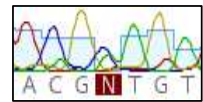

position 2 (nt 1514)

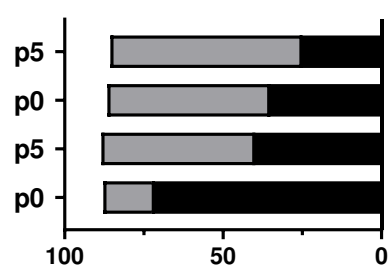

percent of lineage 5 and lineage 3 determined by sanger sequencing
Position 3 (nt 25768)

Single infection
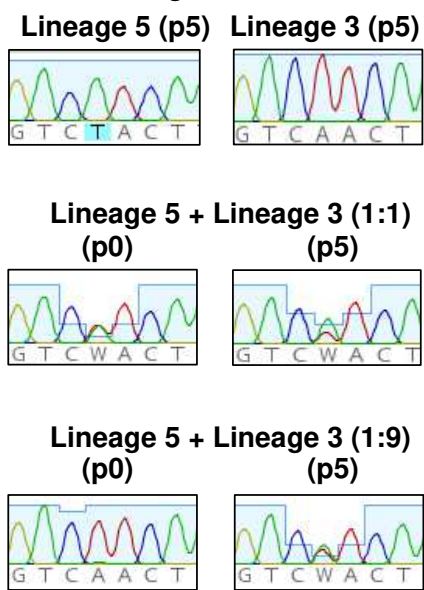

position 3 (nt 25768)

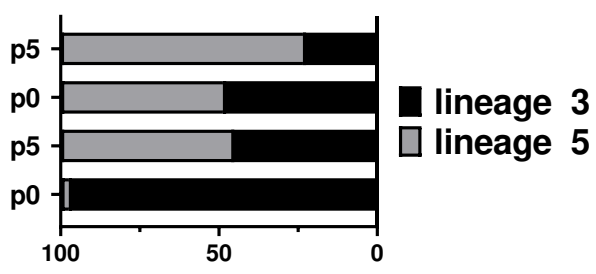

percent of lineage 5 and lineage 3 determined by sanger sequencing
Figure 3: Lineage 5 outcompetes a parental virus strain of lineage 3 in an in-vitro fitness competition assay. Calu- 3 cells were infected in duplicates with 10,000 PFU containing the indicated ratios of a lineage 3 : lineage 5 virus isolate at the time of initial infection (p0). Ca 1000-10000 PFU were transferred in 5 subsequent passages. A) At p0 and p5, viral RNA was isolated from the inoculum/supernatant and two RT-PCRs over two SNP that discriminate lineage 3 and lineage 5 were performed. RT-PCR amplicons were subjected to Sanger sequencing over the respective SNPs to investigate changes in sequencing chromatograms. Data of one representative experiment is shown B) the average peak heights in sequencing chromatograms at each SNP were analyzed using the Chromat Quantitator server and compared between $\mathrm{p} 0$ and $\mathrm{p} 5$ populations. 
A

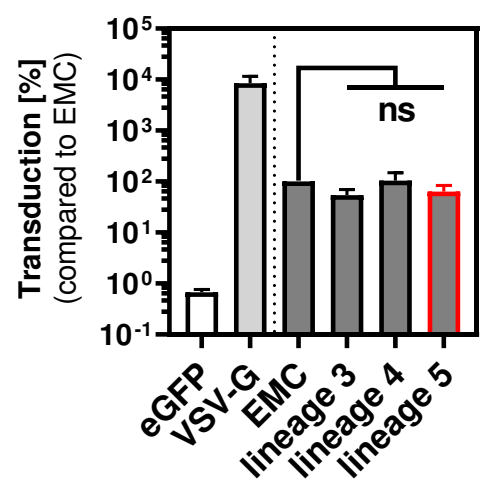

B

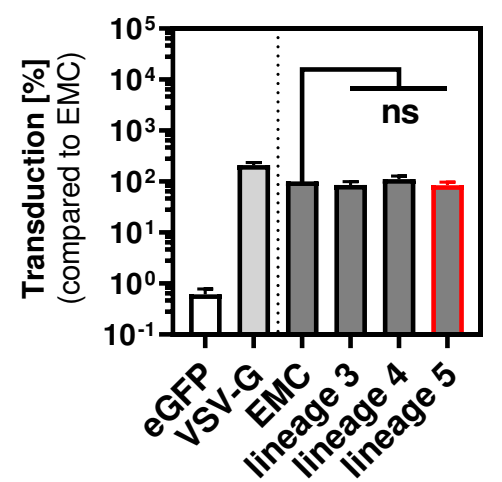

C

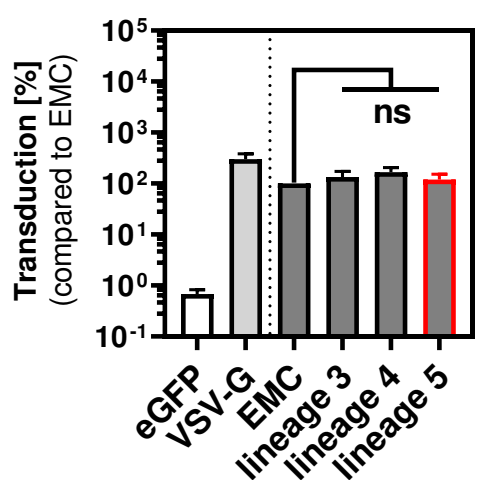

D

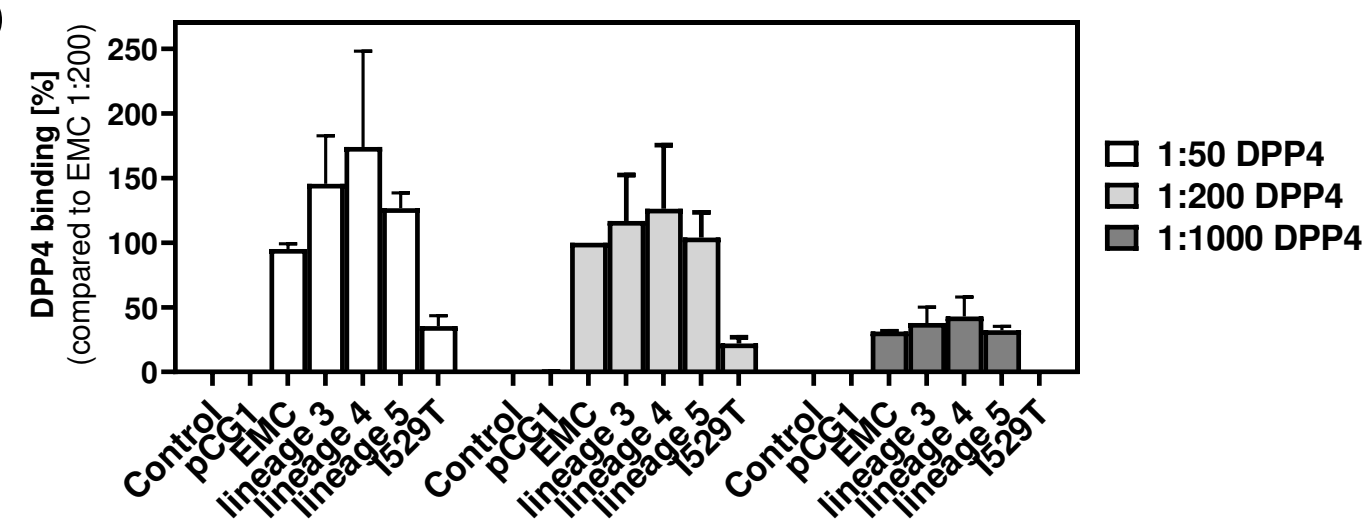

606

Figure 4: lineage specific MERS-CoV spike proteins show no difference in host cell entry and DPP4 binding capacity. A-C) rhabdoviral particles harbouring MERS-CoV spike proteins of the EMC isolate, lineage 3, lineage 4 and lineage 5, VSV-G (positive control), or eGFP (negative control) were inoculated onto TMPRSS2 expressing Calu-3, Caco-2 and TMRPSS2 negative Huh7 cells. Transduction efficiency was quantified at $18 \mathrm{~h}$ post transduction by measuring the activity of virus-encoded luciferase in cell lysates. Transduction mediated by EMC spike protein was set as $100 \%$. The averages from $n=3$ individual experiments performed in quadruplicates are shown; error bars indicate SEMs. Statistical significance was analysed by paired two-tailed Student t tests. D) 293T cells un transfected or transfected to express MERS-CoV lineage specific spike proteins or empty expression vector (pCG1) were detached and incubated with human Fc-tagged, soluble DPP4 (solDPP4-Fc), diluted 1:50, 1:200 and 1:1000 and an Alexa Fluor 488-conjugated anti-human antibody before DPP4 binding was quantified by flow cytometry. For normalization, binding of solDPP4-Fc (1:200) to EMC spike was set as 100\%. For background subtraction of samples incubated with just Alexa Fluor 488-conjugated antibodies (control) was performed for each sample. EMC spike carrying the 1529T SNP was included as an internal control, since this SNP has been previously shown to reduce DPP4 binding [19]. The results of a single representative experiment carried out with triplicate samples are shown and were confirmed in a separate experiment. Error bars indicate SDs. 


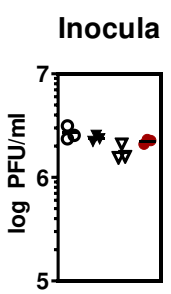

626

\section{7}

628

629

630

631

632

633

634

635

636

637

638

639
ORF1a quantification

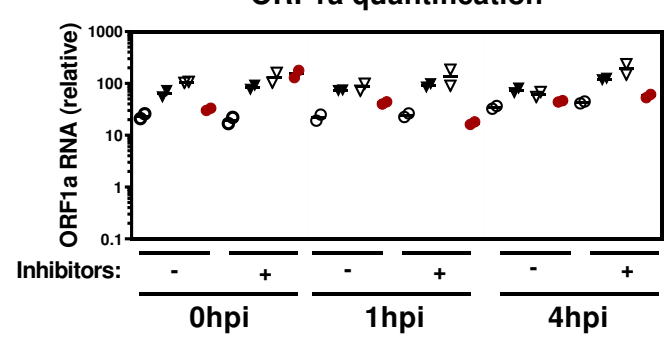

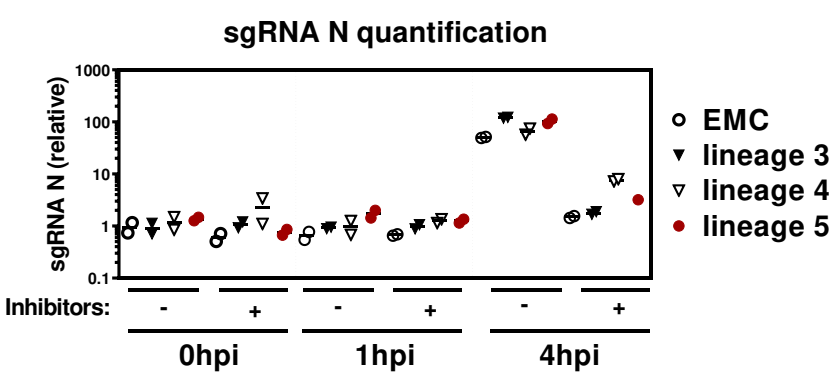

Figure 5. All tested MERS-CoV strains show similar host cell entry. Calu-3 cells were infected in duplicates with one virus isolate of all each phylogenetic lineage (EMC, lineage 3 , lineage 4 and lineage 5) at $\mathrm{MOI}=1$ in the presence or absence of CoV entry inhibitors. Entry inhibited samples were pre-incubated for 1 hour with a cocktail of $25 \mu \mathrm{M}$ Cathepsin $\mathrm{L}$ inhibitor, $25 \mu \mathrm{M}$ Pitstop II, and 100 $\mathrm{M}$ Camostat. The inhibitor cocktail remained on the cells during the whole course of infection. To allow for a synchronized virus entry, virus attachment was performed at $4^{\circ} \mathrm{C}$ for 1 hour, followed by four washing steps with PBS. After virus attachment, infected Calu-3 cells were either immediately lysed (0hpi), or incubated at $37^{\circ} \mathrm{C}$ for 1 hour or 4 hours, respectively ( $1 \mathrm{hpi}$ and $4 \mathrm{hpi}$, respectively). Total RNA was isolated from lysed cells and viral genomic RNA (ORF1a) and subgenomic nucleocapsid mRNA (sgRNA N) was quantified by real-time $\mathrm{qPCR}$ and plotted relative to the amount of the housekeeping gene TBP. The inocula of each phylogenetic lineage were back-titrated by plaque assay to confirm that highly similar amounts of infectious particles were used to infect the cells. 


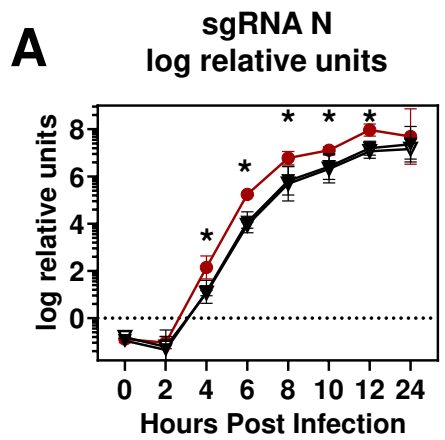

\section{B $\quad$ SgRNA N}

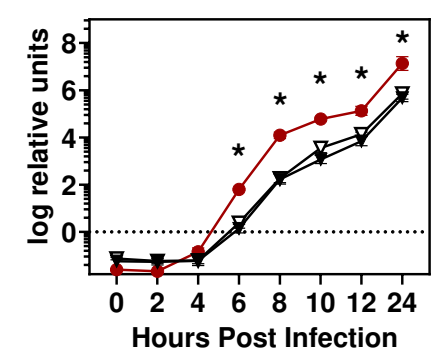

ORF1a RNA log relative units

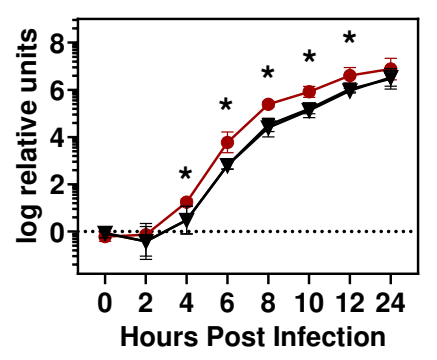

ORF1a RNA

log relative units

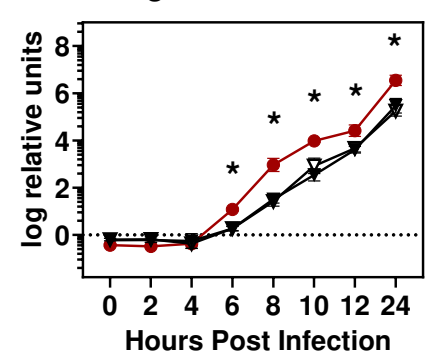

Infectious particles $\log \mathrm{PFU} / \mathrm{ml}$

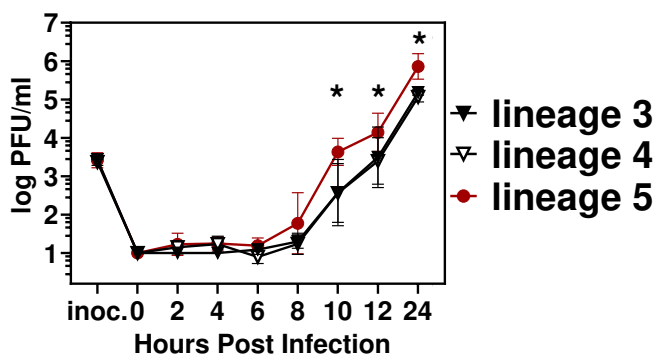

Infectious particles log PFU/ml

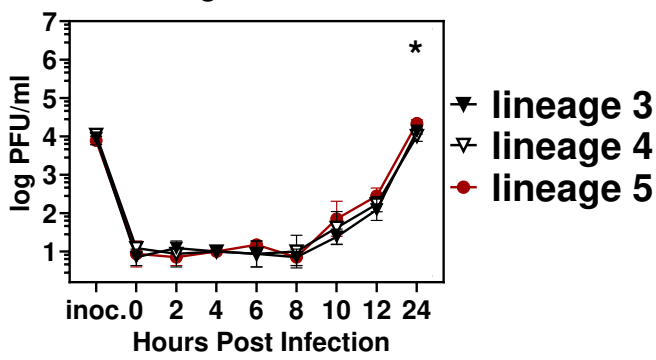

641

642

643

644

645

646

647

648

649

650
Figure 6. Subgenomic nucleocapsid RNA, ORF1a and PFU quantification at early stages

of infection. Calu-3 and Vero B4 cells were infected at an moi of 0.002 with one virus isolate of each phylogenetic lineage in duplicates for each indicated time point in $n=2$ independent experiments. At the indicated time points, cell culture supernatant was harvested and subjected to virus quantification by plaque assay. Infected cells were lysed and total RNA was isolated to be subjected to quantification by real-time PCR of sgRNA N and ORF1a RNA. Cycle thresholds were normalized to the housekeeping gene TBP and the amount of RNA at Ohpi. Statistical significance of differences in virus replication was analyzed by Mann-Whitney test ( $p$-value $<0.0332 ;^{*}$ ). 

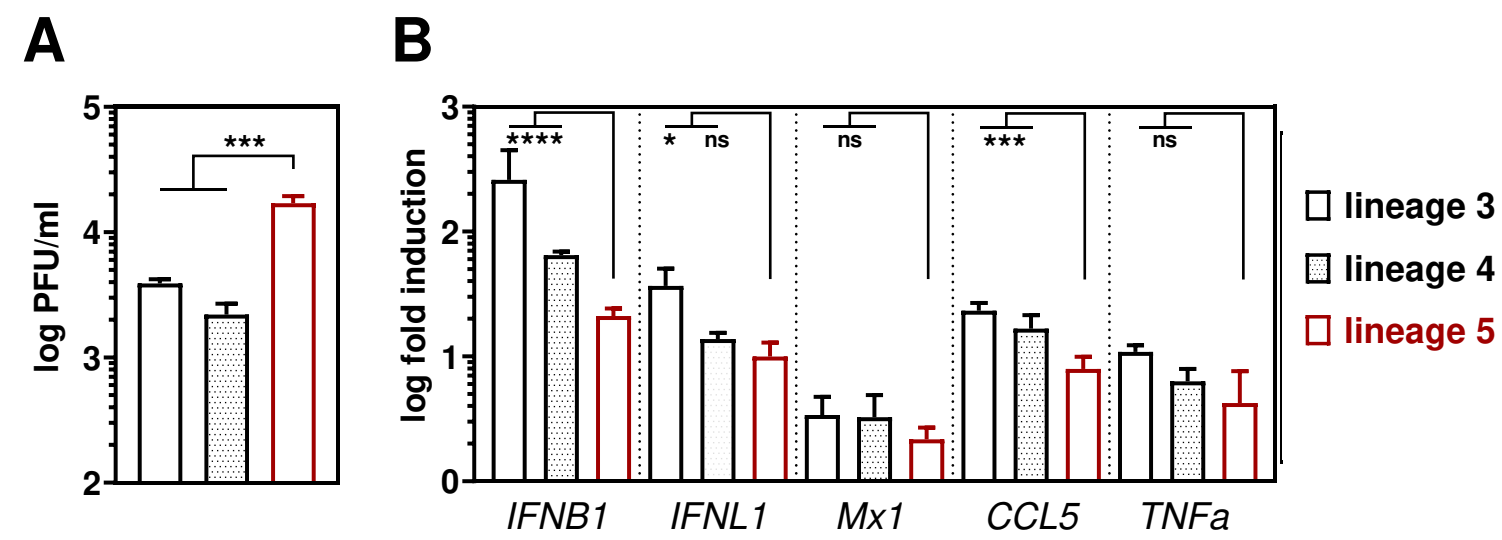

652 Figure 7. Lineage 5 MERS-CoV strains show reduced immune gene induction. Calu-3 653 cells were infected in duplicates with $n=2$ isolates of each phylogenetic lineage with a high 654 multiplicity of infection $(\mathrm{MOI}=2$ ) for subsequent quantitative real-time PCR (q-RT-PCR) 655 analysis of key immune genes. The average mRNA inductions of duplicates of $n=2$ 656 experiments is shown. A) infectious virus production as quantified by plaque assay 12 hours 657 post infection (hpi). B) immune gene induction as quantified by real-time RT-PCR on lysed 658 Calu-3 cells $12 \mathrm{hpi}$, expressed as fold induction over the non-infected control cells, normalized 659 to the housekeeping gene TBP. Statistical significance of differences in virus replication was 660 analyzed by Mann-Whitney test ( $\left.p>0.05, n s ; p \leq 0.05,{ }^{*} ; p \leq 0.01,{ }^{* *} ; p \leq 0.001,{ }^{* * *}\right)$. 


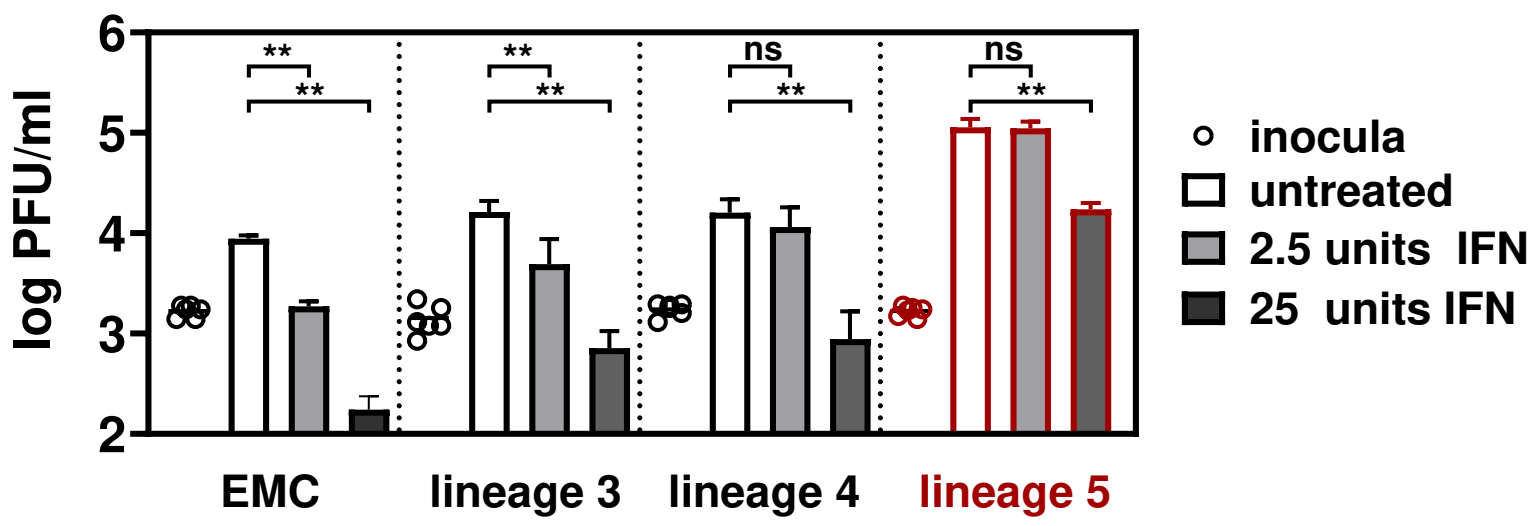

663 Figure 8. Lineage 5 MERS-CoV isolates show decreased IFN sensitivity. Calu-3 cells were 664 incubated for 16 hours with 2.5 or 25 units type-I interferon (IFN) prior to infection at $\mathrm{MOI}=$ 6650.002 with two MERS-CoV isolates of each indicated lineage in triplicates. Inocula, as well as 666 virus progeny in supernatant 24 hours post infection were quantified by plaque assay. Shown 667 are the combined data of two independent experiments with error bars indicating the 668 SD. Statistical significance of differences in virus replication was analyzed by Mann-Whitney 669 test (ns, $\left.\mathrm{P}>0.1234 ;{ }^{* *} \mathrm{P} \leq 0.0021\right)$. 


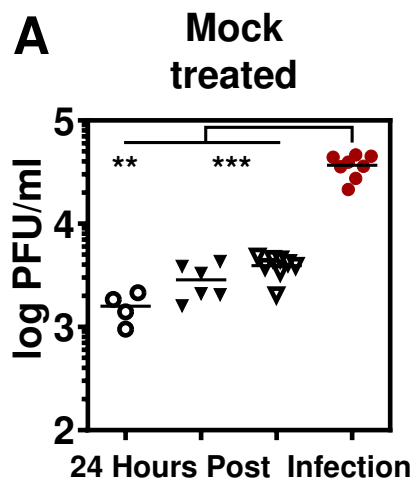

B T84 WT

cells

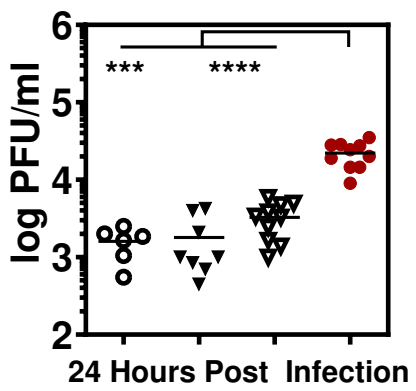

JAK STAT inhibitor

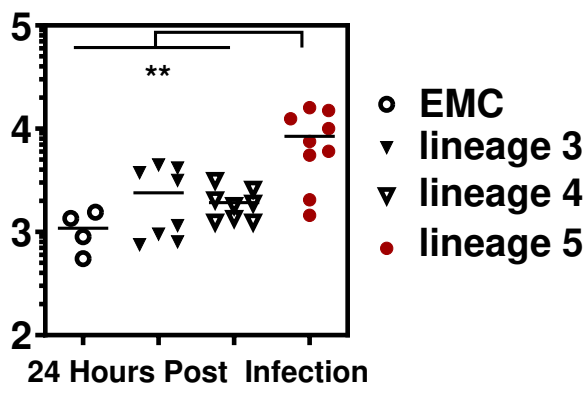

T84 IFNA/LR

KO cells

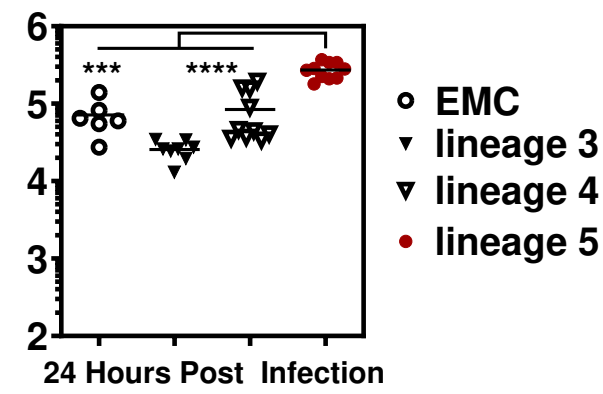

Figure 9: Replication of MERS-CoV isolates in JAK/STAT signalling inhibited and IFN receptor knock-out cells. Higher replication of lineage $5 \mathrm{MERS-CoV}$ is additionally linked to 673 IFN action via the JAK/STAT signaling pathway. A) Calu-3 cells were treated with $50 \mathrm{nM}$ of the 674 JAK/STAT inhibitor Ruxolitinib 1 hour prior and during the infection with two viruses per 675 phylogenetic lineage (lineage 3 , lineage 4 and lineage 5) in duplicates. Virus progeny in the 676 supernatant was quantified by plaque assay at 24hpi. Statistical significance of differences in 677 virus replication was analyzed by Mann-Whitney test $\left({ }^{* *} P \leq 0.0021,{ }^{* * *} P \leq 0.0002\right)$. B) T84 wt 678 and type I and III IFN receptor knock-out cells (IFNAR/IFNLR KO cells) were infected in 679 triplicates. Virus progeny in the supernatant was quantified by plaque assay at $24 \mathrm{hpi}$. Statistical 680 significance of differences in virus replication was analyzed by Mann-Whitney test ${ }^{* \star *} \mathrm{P} \leq$ $\left.6810.0002 ;{ }^{* * *} \mathrm{P} \leq 0.0001\right)$. 
A

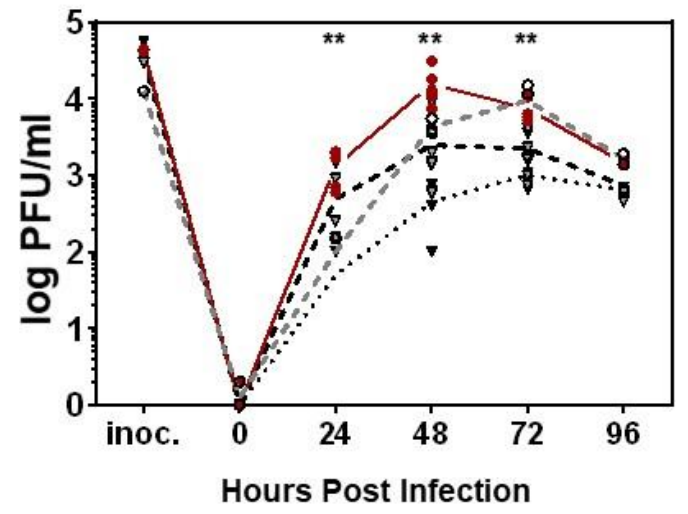

C
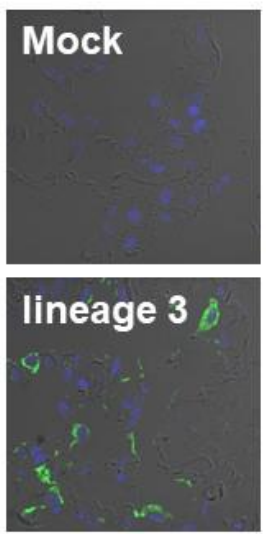
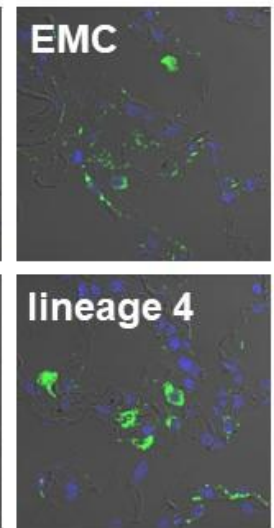

B

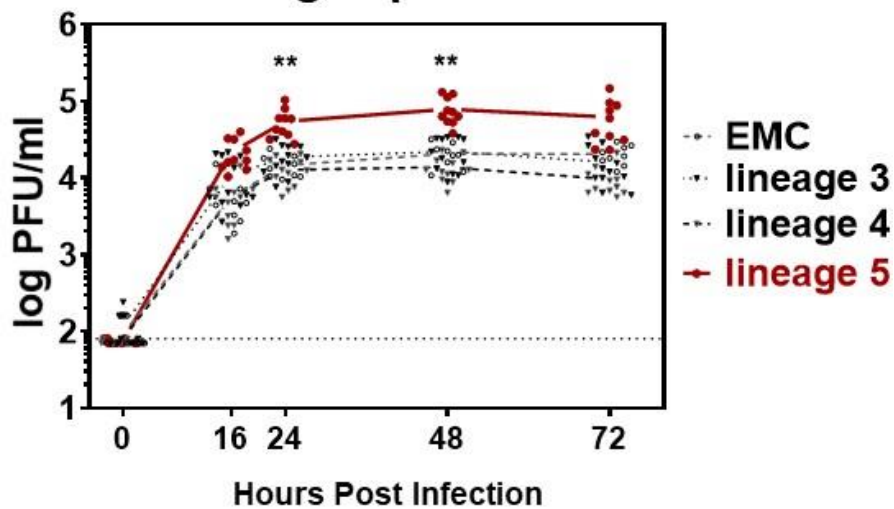

682

683

684

685

686

687

688

689

690

691

692

693

694

695

696

697

698

699

700

701

Figure 10. Growth kinetics of MERS-CoV isolates in models of the human respiratory

tract. A) replication on primary human airway epithelium (HAE) of a single donor. Two isolates of each phylogenetic lineage were used for infection in triplicates. Virus progeny in apical washes was quantified by plaque assay every 24 hours by plaque assay. Statistical significance in difference of PFU/ml between lineage and other lineages was determined by two-tailed Mann-Whitney test $\left({ }^{*} \mathrm{P}<0.0021\right)$. Statistical significant differences in PFU/ml were found at $24 \mathrm{hpi}$ for lineage 5 between lineage $3\left(^{* *}\right)$ and EMC $\left({ }^{*}\right)$; at 48hpi between lineage 5 and lineage $3\left(^{* *}\right)$, lineage $4\left(^{* *}\right)$ and EMC $\left({ }^{*}\right)$; at $72 \mathrm{hpi} \mathrm{between} \mathrm{lineage} 5$ and lineage $3\left(^{*}\right)$ and lineage $\left.4{ }^{* *}\right)$. B) Growth kinetics of MERS-CoV isolates on ex vivo lung explants, derived from three different patients that have undergone lung resection. One isolate of each phylogenetic lineage was used for infection in triplicate for each explant and virus progeny in the supernatant was quantified by plaque assay. Differences in PFU/ml between lineage 5 and other lineages was tested for significance using Krustall-Wallis test $\left({ }^{*} P<0.0021\right)$. C) Immunofluorescent analysis of MERS-CoV lineages replication in ex vivo human lung tisse. Explants from three different patients were infected with one MERS-CoV isolate of each lineage for $24 \mathrm{~h}$. Histological sections were probed with MERS-CoV nucleocapsid antibody (green), nuclei were counterstained with DAPI (blue) and tissue structure was visualized by differential interference contrast. Scale bar represent $20 \mu \mathrm{M}$. D) MERS-CoV replication quantified by immunofluorescent analysis. Values represent the area (in $\mu \mathrm{m}^{2}$ ) of positive-infection, 
702 determined by nucleocapsid immunofluorescent signal in $n=3$ infected lung explants at 24 $703 \mathrm{hpi}$, measured in duplicates. The positive-infected area of each respective isolate was 704 normalized to the total lung tissue area and the value obtained for EMC. Statistical significance 705 in quantified infected area was analyzed by Mann-Whitney test (ns, $\left.P>0.1234 ;{ }^{* *} \mathrm{P} \leq 0.0021\right)$. 
708 1. Zaki, A.M., et al., Isolation of a novel coronavirus from a man with pneumonia in 
23. Muth, D., et al., Infectious Middle East Respiratory Syndrome Coronavirus Excretion and Serotype Variability Based on Live Virus Isolates from Patients in Saudi Arabia. J Clin Microbiol, 2015. 53(9): p. 2951-5.

24. Kindler, E., et al., Efficient Replication of the Novel Human Betacoronavirus EMC on Primary Human Epithelium Highlights Its Zoonotic Potential. mBio, 2013. 4(1): p. e00611-12.

25. Thornbrough, J.M., et al., Middle East Respiratory Syndrome Coronavirus NS4b Protein Inhibits Host RNase L Activation. MBio, 2016. 7(2): p. e00258.

26. Kindler, E., et al., Early endonuclease-mediated evasion of RNA sensing ensures efficient coronavirus replication. PLOS Pathogens, 2017. 13(2): p. e1006195.

27. Niemeyer, D., et al., Middle East respiratory syndrome coronavirus accessory protein $4 a$ is a type I interferon antagonist. J Virol, 2013. 87(22): p. 12489-95.

28. Canton, J., et al., MERS-CoV $4 b$ protein interferes with the NF-kappaB-dependent innate immune response during infection. PLoS Pathog, 2018. 14(1): p. e1006838.

29. Fagbo, S.F., et al., Molecular Epidemiology of Hospital Outbreak of Middle East Respiratory Syndrome, Riyadh, Saudi Arabia, 2014. Emerg Infect Dis, 2015. 21(11): p. 1981-8.

30. Domingo, E., Chapter 3 - Darwinian Principles Acting on Highly Mutable Viruses, in Virus as Populations, E. Domingo, Editor. 2016, Academic Press: Boston. p. 73-122.

31. Gierer, S., et al., The Spike Protein of the Emerging Betacoronavirus EMC Uses a Novel Coronavirus Receptor for Entry, Can Be Activated by TMPRSS2, and Is Targeted by Neutralizing Antibodies. Journal of Virology, 2013. 87(10): p. 5502.

32. Shirato, K., M. Kawase, and S. Matsuyama, Middle East Respiratory Syndrome Coronavirus Infection Mediated by the Transmembrane Serine Protease TMPRSS2. Journal of Virology, 2013. 87(23): p. 12552.

33. Zielecki, F., et al., Human cell tropism and innate immune system interactions of human respiratory coronavirus EMC compared to those of severe acute respiratory syndrome coronavirus. J Virol, 2013. 87(9): p. 5300-4.

34. Terada, Y., et al., Emergence of pathogenic coronaviruses in cats by homologous recombination between feline and canine coronaviruses. PLoS One, 2014. 9(9): p. e106534.

35. Zhou, $\mathrm{H}$., et al., Identification of a novel recombinant virulent avian infectious bronchitis virus. Vet Microbiol, 2017. 199: p. 120-127.

36. Domingo, E., Chapter 8 - Quasispecies Dynamics in Disease Prevention and Control, in Virus as Populations, E. Domingo, Editor. 2016, Academic Press: Boston. p. 263297.

37. Chan, R.W.Y., et al., Tropism and replication of Middle East respiratory syndrome coronavirus from dromedary camels in the human respiratory tract: an in-vitro and exvivo study. The Lancet Respiratory Medicine, 2014. 2(10): p. 813-822.

38. Comar, C.E., et al., Antagonism of dsRNA-Induced Innate Immune Pathways by NS4a and NS4b Accessory Proteins during MERS Coronavirus Infection. mBio, 2019. 10(2): p. e00319-19.

39. Dudas, G., et al., MERS-CoV spillover at the camel-human interface. Elife, 2018. 7.

40. Dijkman, R., et al., Isolation and characterization of current human coronavirus strains in primary human epithelial cell cultures reveal differences in target cell tropism. $\mathrm{J}$ Virol, 2013. 87(11): p. 6081-90.

41. Hocke, A.C., et al., Emerging human middle East respiratory syndrome coronavirus causes widespread infection and alveolar damage in human lungs. Am J Respir Crit Care Med, 2013. 188(7): p. 882-6.

42. Peter, A., et al., Localization and pneumococcal alteration of junction proteins in the human alveolar-capillary compartment. Histochem Cell Biol, 2017. 147(6): p. 707719.

43. Berger Rentsch, M. and G. Zimmer, A vesicular stomatitis virus replicon-based bioassay for the rapid and sensitive determination of multi-species type I interferon. PloS one, 2011. 6(10): p. e25858. 
817 44. Corman, V.M., et al., Detection of a novel human coronavirus by real-time reverse818 transcription polymerase chain reaction. Euro Surveill, 2012. 17(39).

819 45. Zhou, J., et al., Human intestinal tract serves as an alternative infection route for $820 \quad$ Middle East respiratory syndrome coronavirus. Science Advances, 2017. 3(11): p. $821 \quad$ eaao4966. 


\section{Figures}

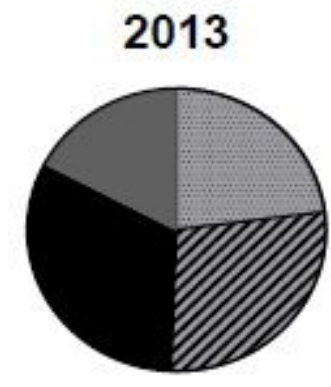

$\mathrm{n}=87$

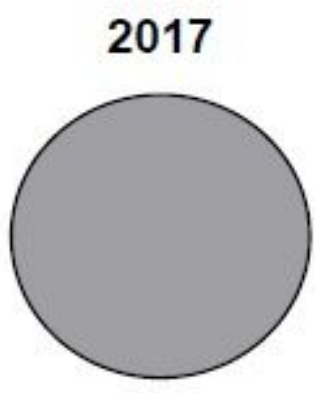

$n=27$

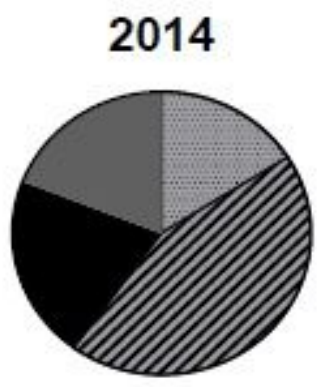

$n=100$

2018

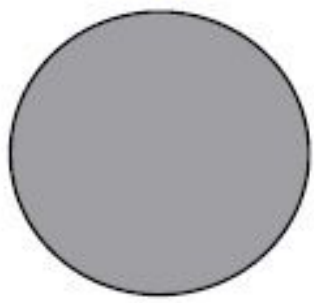

$n=13$

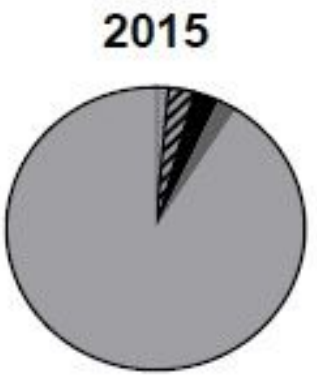

$\mathrm{n}=\mathbf{2 8 7}$

2019

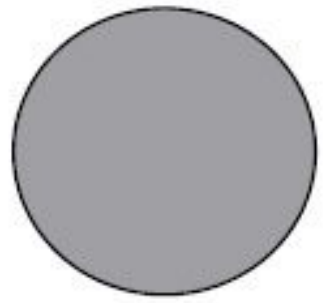

$n=29$

Figure 1

MERS-CoV sequences uploaded to NCBI GenBank between 2013 and 2020. All available GenBank entries for MERS-CoV with a sequence length $\geq 5000$ nucleotides were included $(n=561)$. The collection date provided in GenBank was used for assigning a year to each sequence.

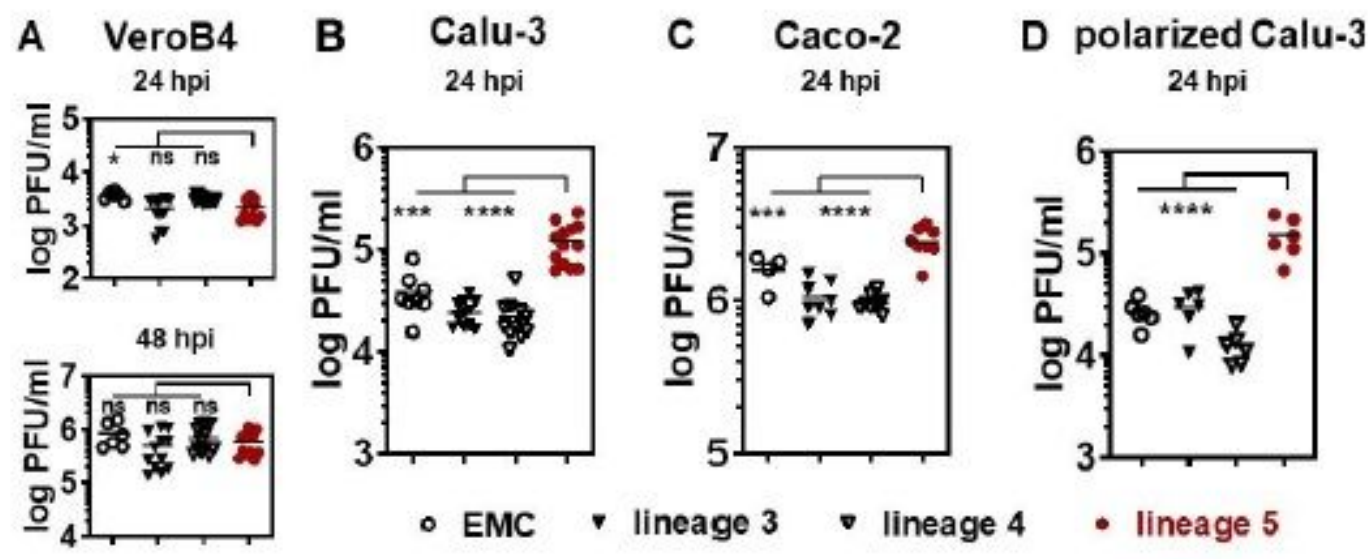

Figure 2

Enhanced replication of lineage 5 virus isolates on permanent and primary cells. Cells were infected at $\mathrm{MOI}=0.002$ and virus progeny in supernatant quantified by plaque assay in cell culture supernatants. A) VeroB4 cells, 24 and 48 hpi; B) Calu-3 lung cells, 24 hpi C) Caco-2 colon cells, 24 hpi; D) Calu-3 lung cells 
grown as air-liquid interface culture, $24 \mathrm{hpi}$. Every dot constitutes the average of triplicate infections performed in $n=4$ (Calu-3), $n=3$ (polarized Calu-3), or $n=2$ independent experiments (Caco-2, VeroB4 and polarized Calu-3 cells) with $n=3$ virus isolates per lineage. Statistical significance in difference of $\mathrm{PFU} / \mathrm{ml}$ between lineage 5 and other lineages was determined by Krustall-Wallis test in $A)\left({ }^{*} P>0.0332\right.$, ns $P<0.05)$, and by two-tailed t-test in $B), C)$ and $D)(* * P<0.0021 ; * \star * P<0.0002$; $* \star \star * P<0.0001)$

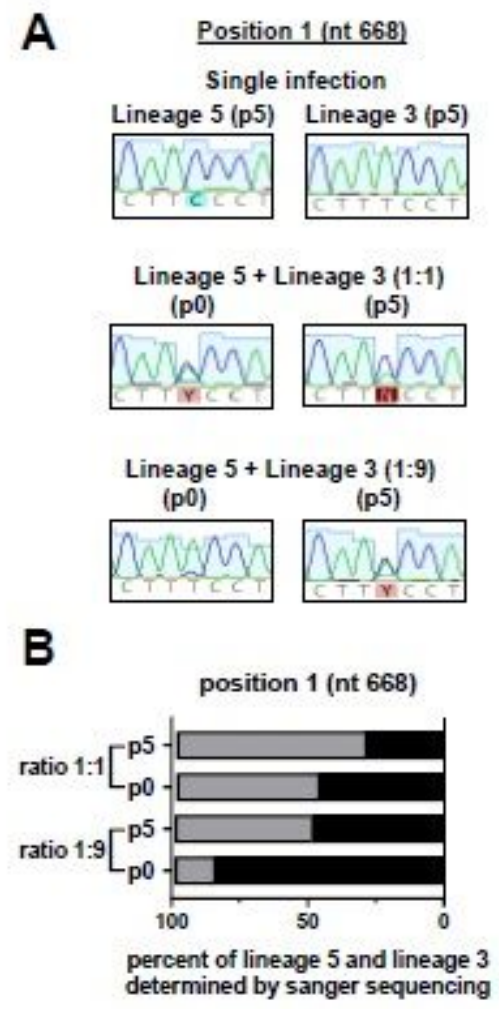

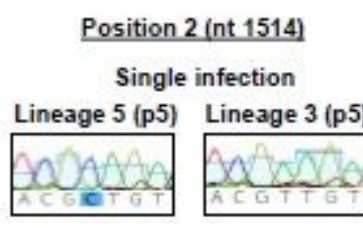
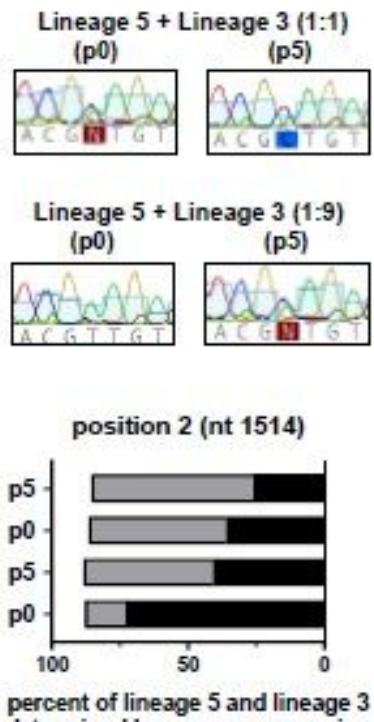

percent of lineage 5 and lineage 3 determined by sanger sequencing
Position 3 (nt 25768)

Single infection

Lineage 5 (p5) Lineage 3 (p5)
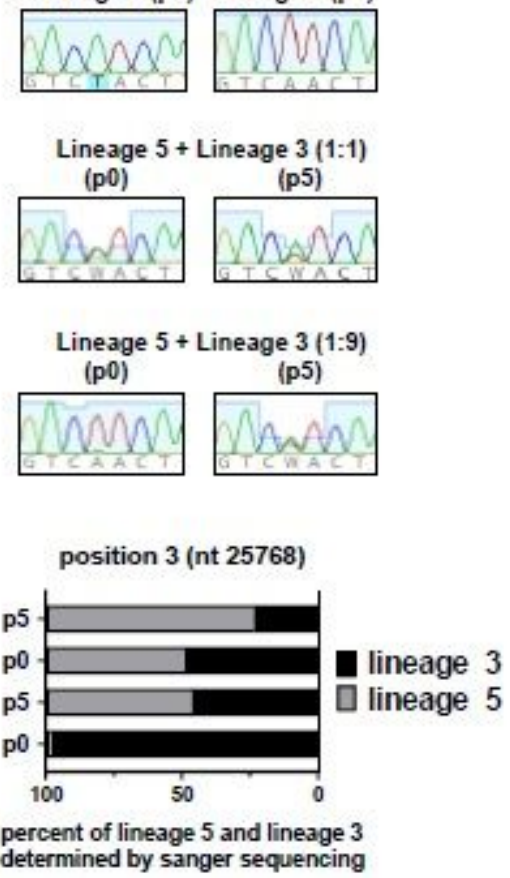

\section{Figure 3}

Lineage 5 outcompetes a parental virus strain of lineage 3 in an in-vitro fitness competition assay. Calu-3 cells were infected in duplicates with 10,000 PFU containing the indicated ratios of a lineage 3 : lineage 5 virus isolate at the time of initial infection (p0). Ca 1000-10000 PFU were transferred in 5 subsequent passages. A) At $\mathrm{p} 0$ and p5, viral RNA was isolated from the inoculum/supernatant and two RT-PCRs over two SNP that discriminate lineage 3 and lineage 5 were performed. RT-PCR amplicons were subjected to Sanger sequencing over the respective SNPs to investigate changes in sequencing chromatograms. Data of one representative experiment is shown $\mathrm{B}$ ) the average peak heights in sequencing chromatograms at each SNP were analyzed using the Chromat Quantitator server and compared between p0 and p5 populations. 
A

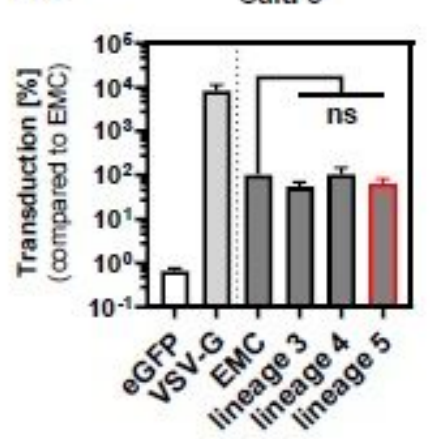

B

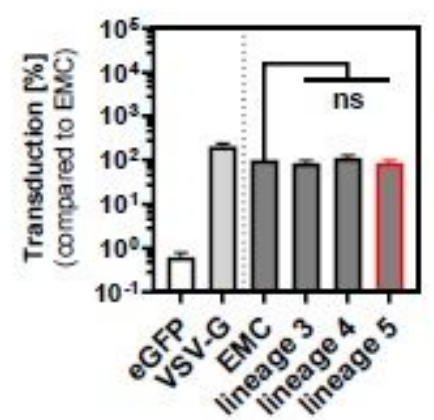

C

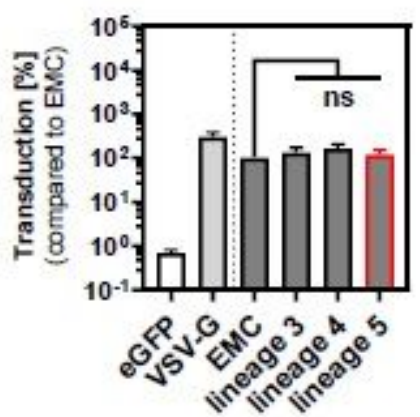

\section{D}

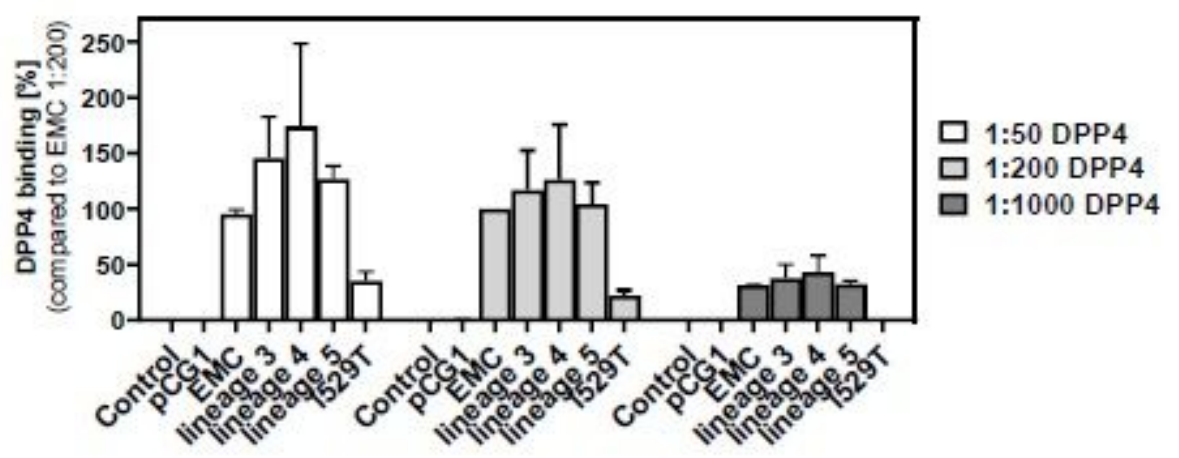

Figure 4

lineage specific MERS-CoV spike proteins show no difference in host cell entry and DPP4 binding capacity. A-C) rhabdoviral particles harbouring MERS-CoV spike proteins of the EMC isolate, lineage 3 , lineage 4 and lineage 5, VSV-G (positive control), or eGFP (negative control) were inoculated onto TMPRSS2 expressing Calu-3, Caco-2 and TMRPSS2 negative Huh7 cells. Transduction efficiency was quantified at $18 \mathrm{~h}$ post transduction by measuring the activity of virus-encoded luciferase in cell lysates. Transduction mediated by EMC spike protein was set as $100 \%$. The averages from $n=3$ individual experiments performed in quadruplicates are shown; error bars indicate SEMs. Statistical significance wasanalysed by paired two-tailed Student t tests. D) 293T cells un transfected or transfected to express MERS-CoV lineage specific spike proteins or empty expression vector (pCG1) were detached and incubated with human Fc-tagged, soluble DPP4 (solDPP4-Fc), diluted 1:50, 1:200 and 1:1000 and an Alexa Fluor 488-conjugated anti-human antibody before DPP4 binding was quantified by flow cytometry. For normalization, binding of solDPP4-Fc (1:200) to EMC spike was set as $100 \%$. For background subtraction of samples incubated with just Alexa Fluor 488-conjugated antibodies (control) was performed for each sample. EMC spike carrying he I529T SNP was included as an internal control, since this SNP has been previously shown to reduce DPP4 binding [19]. The results of a single representative experiment carried out with triplicate samples are shown and were confirmed in a separate experiment. Error bars indicate SDs. ControlpCG1EMClineage 3lineage 4lineage 5I529TControlpCG1EMClineage 3lineage 4lineage 5I529TControlpCG1EMClineage 3lineage 4lineage 5I529T050100150200250DPP4 binding [\%](compared to EMC 1:200)1:50 DPP41:200 DPP41:1000 DPP4eGFPVSV-GEMClineage 3lineage 4lineage 510-1100101102103104105Calu-3Transduction [\%](compared to EMC)nseGFPVSVGEMClineage 3lineage 4lineage 510-1100101102103104105Caco-2Transduction [\%](compared to 

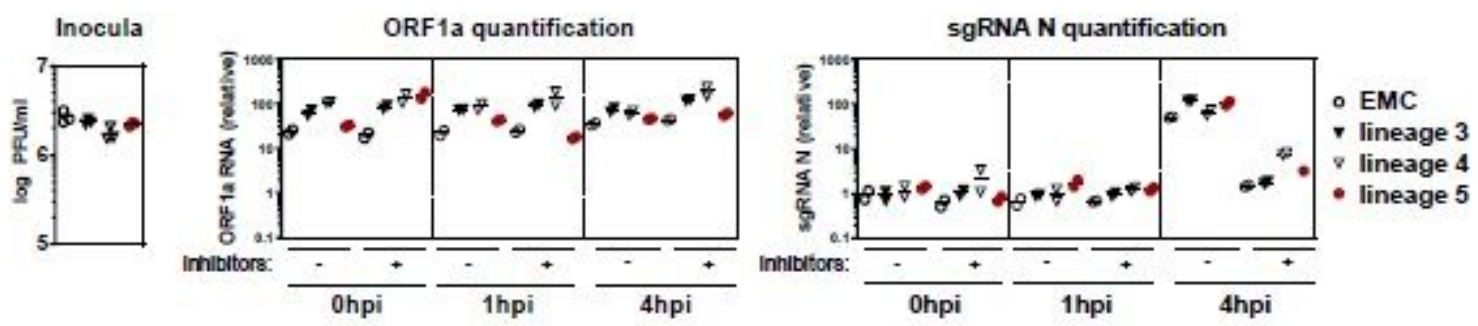

Figure 5

All tested MERS-CoV strains show similar host cell entry. Calu-3 cells were infected in duplicates with one virus isolate of all each phylogenetic lineage (EMC, lineage 3 , lineage 4 and lineage 5 ) at $\mathrm{MOI}=1$ in the presence or absence of CoV entry inhibitors. Entry inhibited samples were pre-incubated for 1 hour with a cocktail of $25 \mu \mathrm{M}$ Cathepsin L inhibitor, $25 \mu \mathrm{M}$ Pitstop II, and $100 \mu \mathrm{M}$ Camostat. The inhibitor cocktail remained on the cells during the whole course of infection. To allow for a synchronized virus entry, virus attachment was performed at $4^{\circ} \mathrm{C}$ for 1 hour, followed by four washing steps with PBS. After virus attachment,infected Calu-3 cells were either immediately lysed (Ohpi), or incubated at $37^{\circ} \mathrm{C}$ for 1 hour or 4 hours, respectively ( $1 \mathrm{hpi}$ and $4 \mathrm{hpi}$, respectively). Total RNA was isolated from lysed cells and viral genomic RNA (ORF1a) and subgenomic nucleocapsid mRNA (sgRNA N) was quantified by real-time qPCR and plotted relative to the amount of the housekeeping gene TBP. The inocula of each phylogenetic lineage were back-titrated by plaque assay to confirm that highly similar amounts of infectious particles were used to infect the cells.
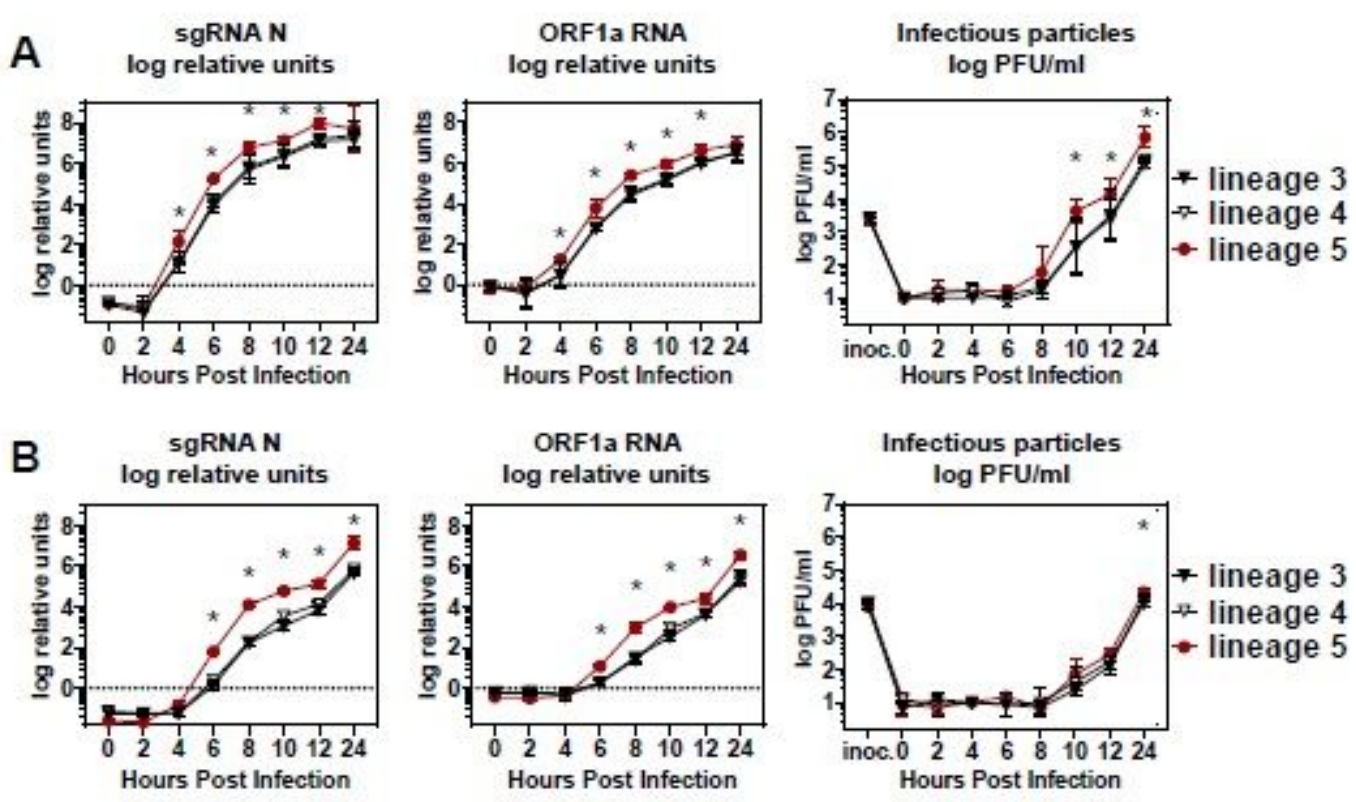

Figure 6 
Subgenomic nucleocapsid RNA, ORF1a and PFU quantification at early stagesof infection. Calu-3 and Vero B4 cells were infected at an moi of 0.002 with one virus isolate of each phylogenetic lineage in duplicates for each indicated time point in $n=2$ independent experiments. At the indicated time points, cell culture supernatant was harvested and subjected to virus quantification by plaque assay. Infected cells were lysed and total RNA was isolated to be subjected to quantification by real-time PCR of sgRNA N and ORF1a RNA. Cycle thresholds were normalized to the housekeeping gene TBP and the amount of RNA at Ohpi. Statistical significance of differences in virus replication was analyzed by Mann-Whitney test (p-value $<0.0332 ; *)$.

A

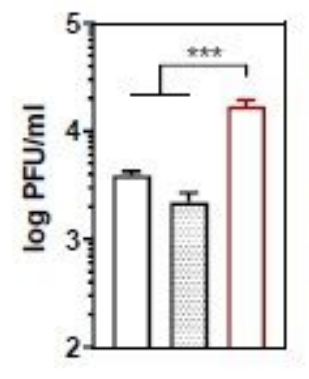

B

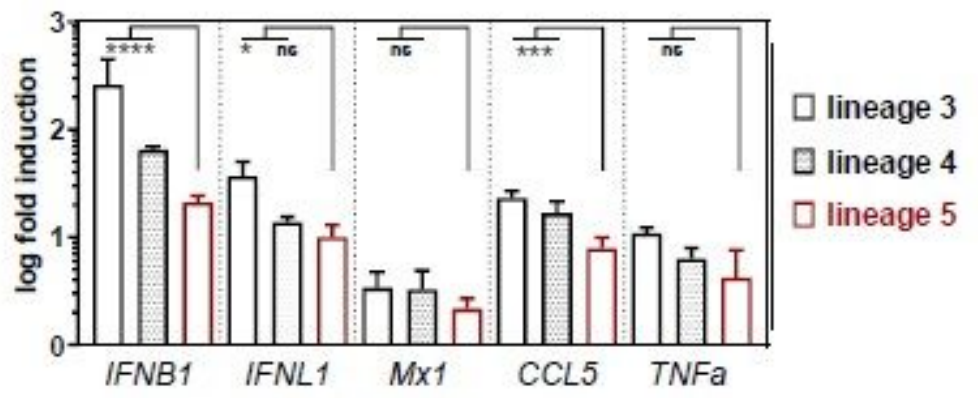

Figure 7

Lineage 5 MERS-CoV strains show reduced immune gene induction. Calu-3 cells were infected in duplicates with $n=2$ isolates of each phylogenetic lineage with a high multiplicity of infection $(\mathrm{MOI}=2)$ for subsequent quantitative real-time PCR (q-RT-PCR) analysis of key immune genes. The average mRNA inductions of duplicates of $n=2$ experiments is shown. A) infectious virus production as quantified by plaque assay 12 hours post infection (hpi). B) immune gene induction as quantified by real-time RT-PCR on lysed Calu-3 cells $12 \mathrm{hpi}$, expressed as fold induction over the non-infected control cells, normalized to the housekeeping gene TBP. Statistical significance of differences in virus replication was analyzed by Mann-Whitney test $(p>0.05, n s ; p \leq 0.05, * ; p \leq 0.01, * * ; p \leq 0.001, * \star *)$.

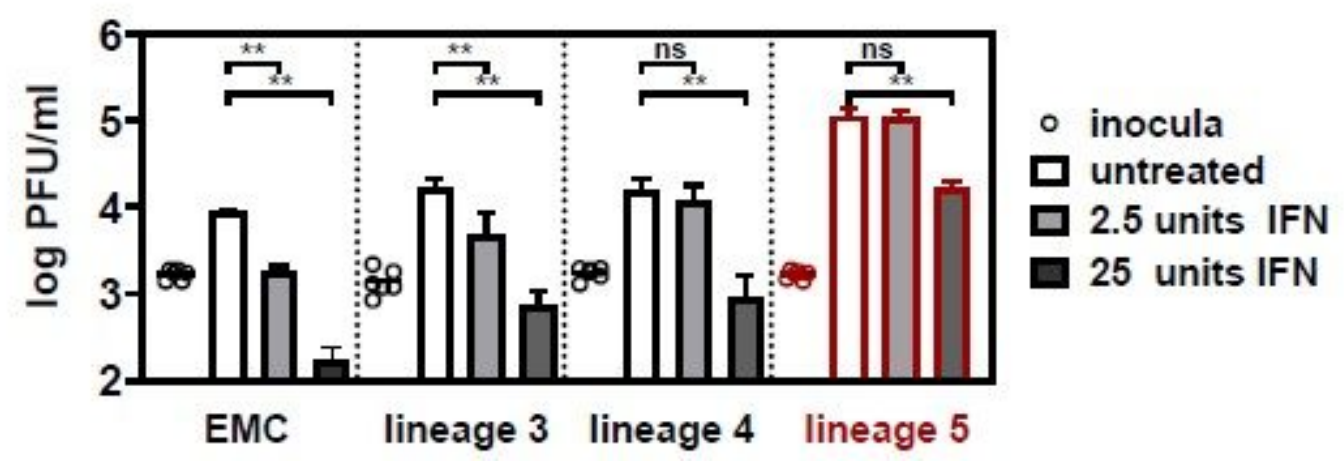

Figure 8

Lineage 5 MERS-CoV isolates show decreased IFN sensitivity. Calu-3 cells were incubated for 16 hours with 2.5 or 25 units type-I interferon (IFN) prior to infection at $\mathrm{MOI}=0.002$ with two MERS-CoV isolates of 
each indicated lineage in triplicates. Inocula, as well as virus progeny in supernatant 24 hours post infection were quantified by plaque assay. Shown are the combined data of two independent experiments with error bars indicating the SD. Statistical significance of differences in virus replication was analyzed by Mann-Whitney test (ns, $\mathrm{P}>0.1234$; ** $\mathrm{P} \leq 0.0021)$.
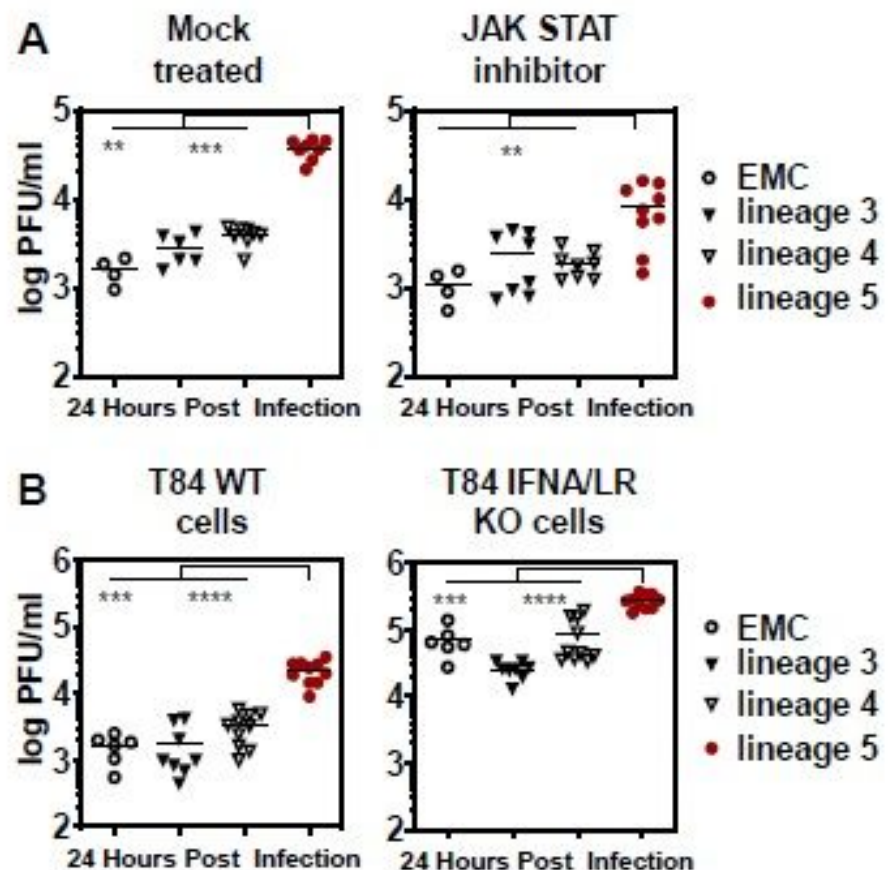

T84 IFNAILR

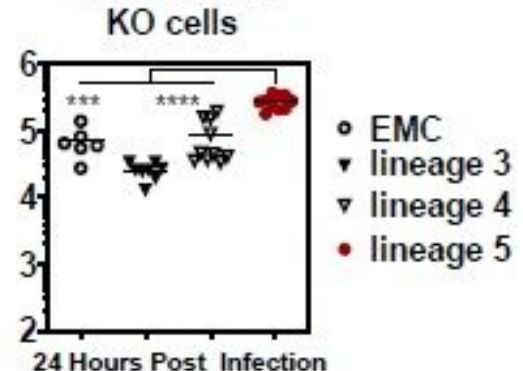

Figure 9

Replication of MERS-CoV isolates in JAK/STAT signalling inhibited and IFN receptor knock-out cells. Higher replication of lineage 5 MERS-CoV is additionally linked to IFN action via the JAK/STAT signaling pathway. A) Calu-3 cells were treated with $50 \mathrm{nM}$ of the JAK/STAT inhibitor Ruxolitinib 1 hour prior and during the infection with two viruses per phylogenetic lineage (lineage 3 , lineage 4 and lineage 5) in duplicates. Virus progeny in the supernatant was quantified by plaque assay at $24 \mathrm{hpi}$. Statistical significance of differences in virus replication was analyzed by Mann-Whitney test $\left({ }^{\star} P \leq 0.0021, * \star * P \leq\right.$ 0.0002). B) T84 wt and type I and III IFN receptor knock-out cells (IFNAR/IFNLR KO cells) were infected in triplicates. Virus progeny in the supernatant was quantified by plaque assay at $24 \mathrm{hpi}$. Statistical significance of differences in virus replication was analyzed by Mann-Whitney test ( ${ }^{\star \star *} \mathrm{P} \leq 6800.0002$; $\star \star \star \star P \leq 0.0001)$. 

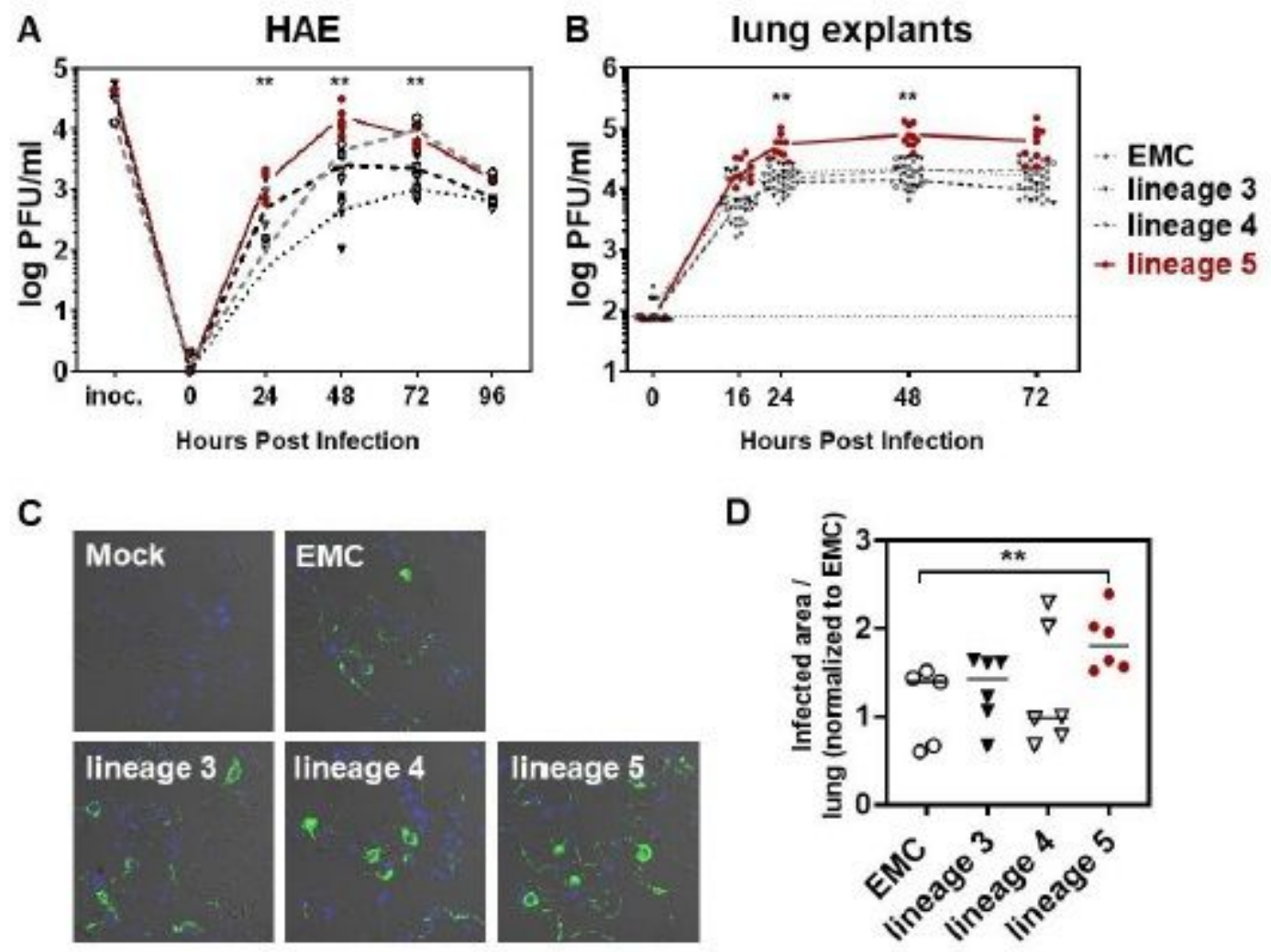

\section{Figure 10}

Growth kinetics of MERS-CoV isolates in models of the human respiratory tract. A) replication on primary human airway epithelium (HAE) of a single donor. Two isolates of each phylogenetic lineage were used for infection in triplicates. Virus progeny in apical washes was quantified by plaque assay every 24 hours by plaque assay. Statistical significance in difference of PFU/ml between lineage and other lineages was determined by two-tailed Mann-Whitney test ( $* \star \mathrm{P}<0.0021)$. Statistical significant differences in PFU/ml were found at $24 \mathrm{hpi}$ for lineage 5 between lineage $3(* *)$ and EMC (*); at $48 \mathrm{hpi}$ between lineage 5 and lineage $3\left(^{* *}\right)$, lineage $4\left(^{* *}\right)$ and EMC $\left({ }^{*}\right)$; at $72 \mathrm{hpi}$ between lineage 5 and lineage $3\left({ }^{*}\right)$ and lineage $4\left(^{* *}\right)$. B) Growth kinetics of MERS-CoV isolates on ex vivo lung explants, derived from three different patients that have undergone lung resection. One isolate of each phylogenetic lineage was used for infection in triplicate for each explant and virus progeny in the supernatant was quantified by plaque assay. Differences in PFU/ml between lineage 5 and other lineages was tested for significance using KrustallWallis test (**P< 0.0021). C) Immunofluorescent analysis of MERS-CoV lineages replication in ex vivo human lung tisse. Explants from three different patients were infected with one MERS-CoV isolate of each lineage for $24 \mathrm{~h}$. Histological sections were probed with MERS-CoV nucleocapsid antibody (green), nuclei were counterstained with DAPI (blue) and tissue structure was visualized by differential interference contrast. Scale bar represent $20 \mu \mathrm{M}$. D) MERS-CoV replication quantified by immunofluorescent analysis. Values represent the area (in $\mu \mathrm{m} 2$ ) of positive-infection, determined by nucleocapsid immunofluorescent signal in $n=3$ infected lung explants at $24 \mathrm{hpi}$, measured in duplicates. The positive-infected area of each respective isolate was normalized to the total lung tissue area and the 
value obtained for EMC. Statistical significance in quantified infected area was analyzed by MannWhitney test (ns, $\mathrm{P}>0.1234 ; * \star \mathrm{P} \leq 0.0021)$. 